\title{
Sequential Posted-Price Mechanisms with Correlated Valuations
}

\author{
MAREK ADAMCZYK, University of Warsaw \\ ALLAN BORODIN, University of Toronto \\ DIODATO FERRAIOLI, University of Salerno \\ BART DE KEIJZER, Centrum Wiskunde \& Informatica (CWI) \\ STEFANO LEONARDI, Sapienza University of Rome
}

\begin{abstract}
We study the revenue performance of sequential posted-price mechanisms and some natural extensions for a setting where the valuations of the buyers are drawn from a correlated distribution. Sequential posted-price mechanisms are conceptually simple mechanisms that work by proposing a "take-it-or-leave-it" offer to each buyer. We apply sequential posted-price mechanisms to single-parameter multiunit settings in which each buyer demands only one item and the mechanism can assign the service to at most $k$ of the buyers.

For standard sequential posted-price mechanisms, we prove that with the valuation distribution having finite support, no sequential posted-price mechanism can extract a constant fraction of the optimal expected revenue, even with unlimited supply. We extend this result to the case of a continuous valuation distribution when various standard assumptions hold simultaneously (i.e., everywhere-supported, continuous, symmetric, and normalized (conditional) distributions that satisfy regularity, the MHR condition, and affiliation). In fact, it turns out that the best fraction of the optimal revenue that is extractable by a sequential posted-price mechanism is proportional to the ratio of the highest and lowest possible valuation.

We prove that a simple generalization of these mechanisms achieves a better revenue performance; namely, if the sequential posted-price mechanism has for each buyer the option of either proposing an offer or asking the buyer for its valuation, then a $\Omega(1 / \max \{1, d\})$ fraction of the optimal revenue can be extracted, where $d$ denotes the degree of dependence of the valuations, ranging from complete independence $(d=0)$ to arbitrary dependence $(d=n-1)$.
\end{abstract}

CCS Concepts: - Theory of computation $\rightarrow$ Algorithmic mechanism design; Computational pricing and auctions; Approximation algorithms analysis; Online algorithms; $\bullet$ Applied computing $\rightarrow$ Online auctions;

Additional Key Words and Phrases: Mechanism design, posted pricing, approximation, correlated values, interdependence

This work was supported by the EU FET project MULTIPLEX no. 317532, the ERC StG Project PAAl 259515, the Google Research Award for Economics and Market Algorithms, the Italian MIUR PRIN 2010-2011 project ARS TechnoMedia Algorithmics for Social Technological Networks, and by "GNCS-INdAM."

Authors' addresses: M. Adamczyk, University of Warsaw, Institute of Informatics, Banacha 2, Warsaw, 02-097, Poland; email: m.adamczyk@mimuw.edu.pl; A. Borodin, University of Toronto, department of Computer Science, 10 Kings College Road, Toronto, M5S-3G4, Canada; email: bor@cs.toronto.edu; D. Ferraioli, University of Salerno, Dipartimento di Ingegneria dell'Informazione ed Elettrica e Matematica Applicata, Via Giovanni Paolo II, 132, 84084, Fisciano, Italy; email: ferraioli@unisa.it; B. d. Keijzer, Centrum Wiskunde \& Informatica (CWI), Networks \& Optimization Group, Science Park 123, 1098 XG, Amsterdam, The Netherlands; email: keijzer@cwi.nl; S. Leonardi, Sapienza University of Rome, Department of Computer, Control and Management Engineering Antonio Ruberti, Via Ariosto 25, 00185, Rome, Italy; email: leonardi@dis.uniroma1.it.

Permission to make digital or hard copies of all or part of this work for personal or classroom use is granted without fee provided that copies are not made or distributed for profit or commercial advantage and that copies bear this notice and the full citation on the first page. Copyrights for components of this work owned by others than ACM must be honored. Abstracting with credit is permitted. To copy otherwise, or republish, to post on servers or to redistribute to lists, requires prior specific permission and/or a fee. Request permissions from permissions@acm.org.

(C) 2017 ACM 2167-8375/2017/12-ART22 \$15.00

https://doi.org/10.1145/3157085

ACM Transactions on Economics and Computation, Vol. 5, No. 4, Article 22. Publication date: December 2017. 


\section{ACM Reference format:}

Marek Adamczyk, Allan Borodin, Diodato Ferraioli, Bart de Keijzer, and Stefano Leonardi. 2017. Sequential Posted-Price Mechanisms with Correlated Valuations. ACM Trans. Econ. Comput. 5, 4, Article 22 (December 2017), 39 pages.

https://doi.org/10.1145/3157085

\section{INTRODUCTION}

A large body of literature in the field of mechanism design focuses on the design of auctions that are optimal with respect to some given objective function, such as maximizing the social welfare or the auctioneer's revenue. This literature mainly considers direct-revelation mechanisms, in which each buyer submits a bid that represents his or her valuation for getting the service, and the mechanism determines the winners and how much they are forced to pay. The reason for this is the revelation principle (see, e.g., Börgers (2015)), which implies that one may resort to studying only direct-revelation mechanisms for many purposes, such as maximizing the social welfare or the auctioneer's revenue. Some of the most celebrated mechanisms follow this approach, such as the Vickrey-Clark-Groves mechanism (Vickrey 1961; Clarke 1971; Groves 1973) and the Myerson mechanism (Myerson 1981).

A natural assumption behind these mechanisms is that buyers will submit truthfully whenever the utility they take with the truthful bid is at least as high as the utility they may take with a different bid. However, it has often been acknowledged that such an assumption may be too strong in a real-world setting. In particular, Sandholm and Gilpin (2004) highlight that this assumption frequently fails because of (1) a buyer's unwillingness to fully specify his or her values; (2) a buyer's unwillingness to participate in ill-understood, complex, unintuitive auction mechanisms; and (3) irrationality of a buyer, which leads him or her to underbid even when it is known that there is nothing to be gained from this behavior. The failure of direct-revelation mechanisms is also confirmed by experimental studies (see, e.g., Kagel et al. (1987)).

This has recently motivated research about auction mechanisms that are conceptually simple. Among these, the class of sequential posted price mechanisms (Chawla et al. 2010) is particularly attractive. First studied by Sandholm and Gilpin (2004) (and called "take-it-or-leave-it mechanisms"), these mechanisms work by iteratively selecting a buyer that has not been selected previously and offering him or her a price. The buyer may then accept or reject that price. When the buyer accepts, he or she is allocated the service. Otherwise, the mechanism does not allocate the service to the buyer. In the sequential posted-price mechanism, we allow both the choice of buyer and the price offered to that buyer to depend on the decisions of the previously selected buyers (and the prior knowledge about the buyers' valuations). Also, randomization in the choice of the buyer and in the charged price is allowed. Sequential posted price mechanisms are thus conceptually simple and buyers do not have to reveal their valuations for the service. Moreover, they are individually rational; that is, informally, participation in such an auction is never harmful to the buyer. Finally, they possess a trivial dominant strategy: indeed, it is always best for a buyer to respond sincerely to any offer that the mechanism makes him or her. Equivalently, following the definition of $\mathrm{Li}$ (2015), we can say that these mechanisms are obviously strategy proof.

Sequential posted price mechanisms have been mainly studied for the setting where the valuations of the buyers are each drawn independently from publicly known buyer-specific distributions, called the independent private values setting. In this article, we study a much more general setting and assume that the entire vector of valuations is drawn from one publicly known distribution, which allows for arbitrarily complex dependencies among the valuations of the buyers. This setting is commonly known as the correlated private values setting. Our goal is to investigate 
questions related to the existence of sequential posted-price mechanisms that achieve a high revenue. That is, we quantify the quality of a mechanism by comparing its expected revenue to that of the optimal mechanism, defined as the mechanism that achieves the highest expected revenue among all dominant strategy-incentive-compatible and ex-post individually rational mechanisms. (In Section 2, we explain and motivate this optimality notion further.)

We assume a standard Bayesian, transferable, quasi-linear utility model and we study a unit demand, single-parameter, multiunit setting: there is one service (or type of item) being provided by the auctioneer, any buyer is interested in receiving the service (or item) once, and the valuation of each buyer consists of a single number that reflects to what extent a buyer would profit from receiving the service provided by the auctioneer. The auctioneer can charge a price to a bidder, so that the utility of a bidder is his or her valuation (in case he or she gets the service), minus the charged price. We focus in this article on the $k$-limited supply setting, where service can be provided to at most $k$ of the buyers. This is an important setting because it is a natural constraint in many realistic scenarios, and it contains two basic special cases: the unit supply setting (where $k=1$ ) and the unlimited supply setting (where $k=n$ ).

Related work. There has been recent substantial work on the subject of revenue performance for simple mechanisms (Babaioff et al. 2014; Devanur et al. 2015; Hart and Nisan 2012; Hartline and Roughgarden 2009; Rubinstein and Weinberg 2015). In particular, Babaioff et al. (2014) highlight the importance of understanding what is the relative strength of simple versus complex mechanisms with regard to revenue maximization.

As described above, sequential posted-price mechanisms are an example of a simple class of mechanisms. Sandholm and Gilpin (2004) initiated the study of sequential posted-price mechanisms. They provided experimental results for the case in which values are independently drawn from the uniform distribution in $[0,1]$. Moreover, they consider the case where multiple offers can be made to a bidder and study the equilibria that arise from this. Blumrosen and Holenstein (2008) compare fixed-price (called symmetric auctions), sequential posted-price (called discriminatory auctions), and the optimal mechanism for valuations drawn from a wide class of i.i.d. distributions. Babaioff et al. (2012) consider prior-independent posted-price mechanisms with $k$-limited supply for the setting where the only information known about the valuation distribution is that all valuations are independently drawn from the same distribution with support $[0,1]$. Postedprice mechanisms have also been previously studied in Kleinberg and Leighton (2003), Blum and Hartline (2005), and Blum et al. (2004), albeit for a non-Bayesian, online setting. In recent work, Feldman et al. (2015) study "online" posted-price mechanisms for combinatorial auctions when valuations are independently drawn.

The works of Chawla et al. (2010) and Gupta and Nagarajan (2013) are closest in spirit to our present work, although they only consider sequential posted-price mechanisms in the independent values setting. In particular, Chawla et al. (2010) prove that such mechanisms can extract a constant factor of the optimal revenue for single- and multiple-parameter settings under various constraints on the allocations. They also consider order-oblivious (i.e., "online") sequential postedprice mechanisms in which the order of the buyers is fixed and adversarially determined. They use order-oblivious mechanisms in order to establish some results for the more general multiparameter case. Yan (2011) builds on this work and strengthens some of the results of Chawla et al. (2010). Moreover, Kleinberg and Weinberg (2012) and Feldman et al. (2016) prove results that imply a strengthening of some of the results of Chawla et al. (2010).

Gupta and Nagarajan (2013) introduce a more abstract stochastic probing problem that includes Bayesian sequential posted-price mechanisms as well as the stochastic matching problem introduced by Chen et al. (2009). Their approximation bounds were later improved by Adamczyk et al. 
(2014), who in particular matched the approximation of Chawla et al. (2010) for the case where there is a single matroid feasibility constraint.

In this work, we focus on the correlated setting. There is now a substantial literature that studies computational complexity and develops mechanisms with good approximation guarantees for revenue maximization in the correlated setting. Many of these mechanisms build on the lookahead mechanism of Ronen (2001) and, as we shall explain, differ from the mechanisms proposed in this work. We will now briefly discuss this body of literature.

The lookahead mechanism of Ronen (2001) and its extension to matroid constraints in Chawla et al. (2014) are fundamental references for the correlated setting and resemble our blind-offer mechanism but are different in substantial ways, as we will discuss below. In Chawla et al. (2014), the GVCG mechanism with lazy reserve prices is proposed (along with some variations thereof), which is shown to 2-approximate the optimal revenue under the right choice of reserve prices for settings more general than the one we study here. More precisely: the approximation factor is achieved for interdependent values satisfying a single crossing property, where items may be allocated according to a matroid constraint. This mechanism is a generalization of the VCG mechanisms with lazy reserves, which was shown by $\mathrm{Li}(2016)$ to attain a $1 / e$-approximation to the optimal revenue in case the distributions are continuous and have a monotone hazard rate.

Cremer and McLean (1988) made a fundamental contribution to auction theory in the correlated value setting by characterizing exactly for which valuation distributions it is possible to extract the full optimal social welfare as revenue. They do this for the ex-post IC, interim IR mechanisms and for the dominant strategy IC, interim IR mechanisms. Related to this is the work of McAfee et al. (1989), which studies a simple mechanism for the extreme case of correlation where all valuations are equal. Moreover, McAfee and Reny (1992) builds on Cremer and McLean (1988) and obtains similar results for more general settings.

The work of Dobzinski et al. (2011) also studies revenue in (predominantly single-item) mechanism design for the setting with correlated values. In particular, they study the performance gap of randomized versus deterministic mechanisms and propose various approximation mechanisms for different models of access to the valuation distribution. Among these mechanisms is an extension of the lookahead mechanism of Ronen (2001), which achieves improved approximation factors. Moreover, it introduces a linear programming representation for expressing the optimal mechanism, which is an idea that we also use in the present article. In Segal (2003), a characterization is given of optimal ex-post incentive compatible and ex-post individually rational optimal mechanisms. Moreover, Roughgarden and Talgam-Cohen (2013) study optimal mechanism design in the more general interdependent setting, and show how to extend the Myerson mechanism to this setting for various assumptions on the valuation distribution. Social welfare maximization for the interdependent setting is studied by Maskin and Dasgupta (2000) and Ausubel (1999), where extensions of the VCG mechanism are presented. Babaioff et al. (2012), Abraham et al. (2013), and Syrgkanis et al. (2015) study various other mechanism design and auction settings with correlated and interdependent valuations.

Lastly, there exists some results regarding the computational complexity of mechanism design with correlated valuations: Ronen and Saberi (2002) prove a polynomial-time inapproximability theorem for ascending auctions, and Papadimitriou and Pierrakos (2011) show that it is NP-hard to compute the optimal deterministic mechanism that is ex-post IC and ex-post IR for three or more buyers. On the positive side, they show that it can be done in polynomial time for the case of two buyers.

Contributions and outline. We define some preliminaries and notation in Section 2. In Section 3, we first present a simple sequence of instances. It shows that for unrestricted correlated 
distributions, the expected revenue of the best sequential posted-price mechanism does not approximate to within any constant factor the expected revenue of the optimal dominant strategy incentive compatible and ex-post individually rational mechanism. This holds for any value of $k$ (i.e., the size of the supply). We extend this impossibility result by proving that a constant approximation is impossible to achieve even when we assume that the valuation distribution is continuous and satisfies all of the following conditions simultaneously: the valuation distribution is supported everywhere, is entirely symmetric, satisfies regularity, satisfies the monotone hazard rate condition, and satisfies affiliation; all the induced marginal distributions have finite expectation; and all the conditional marginal distributions are nonzero everywhere.

The approximation ratio that a sequential posted-price mechanism can generate on our examples is shown to be characterized by the logarithm of the ratio between the highest and lowest valuations in the support of the distribution. It follows from a result by Lavi and Nisan (2004) that this approximation ratio is essentially tight.

Given these negative results, we consider a generalization of sequential posted-price mechanisms that are more suitable for settings with limited dependence among the buyers' valuations: enhanced sequential posted-price mechanisms. An enhanced sequential posted-price mechanism works by iteratively selecting a buyer that has not been selected previously. The auctioneer can either offer the selected buyer a price or ask him or her to report his or her valuation. As in sequential posted-price mechanisms, if the buyer is offered a price, then he may accept or reject that price. When the buyer accepts, he is allocated the service. Otherwise, the mechanism does not allocate the service to the buyer. If instead the buyer is asked to report his or her valuation, then the mechanism does not allocate him or her the service. Our use of enhanced sequential posted-price mechanisms requires that some fraction of buyers reveal their valuation truthfully. Thus, the original property that the bidders not have to reveal their preferences is partially sacrificed, in return for a more powerful class of mechanisms and (as we will see) a better revenue performance. For practical implementation, such mechanisms can be slightly adjusted by providing a bidder with a small monetary reward in case he or she is asked to reveal his or her valuation. ${ }^{1}$

For the enhanced sequential posted-price mechanisms, we prove that again there are instances in which the revenue is not within a constant fraction of the optimal revenue. However, we show that this class of mechanisms can extract a fraction $\Theta(1 / n)$ of the optimal revenue, i.e., a fraction that is independent of the valuation distribution.

We highlight that our positive results do not make any assumptions on the marginal valuation distributions of the buyers or the type of correlation among the buyers. An exception is Section 4, where we consider the case in which the degree of dependence among the buyers is limited. In particular, we introduce the notion of $d$-dimensionally dependent distributions. This notion informally requires that for each buyer $i$, there is a set $S_{i}$ of $d$ other buyers such that the distribution of $i$ 's valuation when conditioning on the vector of other buyers' valuations can likewise be obtained by only conditioning on the valuations of $S_{i}$. Thus, this notion induces a hierarchy of $n$ classes of valuation distributions with increasing degrees of dependence among the buyers: for $d=0$, the buyers have independent valuations, while the other extreme $d=n-1$ implies that the valuations are drawn from an arbitrary (correlated) distribution. Note that $d$-dimensional dependence does not require that the marginal valuation distributions of the buyers themselves satisfy any particular property, and neither does it require anything from the

\footnotetext{
${ }^{1}$ We also note that in some realistic scenarios (e.g., online internet shopping), the valuation of some buyers may be known a priori to the auctioneer (through, e.g., the repetition of auctions or accounting and profiling operations). In such settings, where it is not necessary to query such a buyer for his or her valuation, our results for enhanced sequential posted-price mechanisms are also relevant.
} 
type of correlation or dependence that may exist among the buyers. This stands in contrast with common assumptions such as symmetry, affiliation, the monotone-hazard rate assumption, and regularity, which are often encountered in the auction theory and mechanism design literature.

Our main positive result for enhanced sequential posted-price mechanisms then states that when the valuation distribution is $d$-dimensionally dependent, there exists an enhanced sequential posted-price mechanism that extracts an $\Omega(1 / d)$ fraction of the optimal revenue.

In order to prove this result, we consider blind-offer mechanisms: these mechanisms inherit a critical limitation of oblivious (i.e., online) posted-price mechanisms where buyers are considered sequentially and are offered threshold prices. In contrast to sequential posted-price mechanisms, blind-offer mechanisms are direct-revelation mechanisms where now each buyer $i$ bids for obtaining service and gets the service only if the bid is at least the offered threshold price. This threshold price for buyer $i$ is restricted to be a function of the marginal valuation distribution of $i$, which is in turn a function of the vector of bids submitted by all buyers other than $i$. This generalization sacrifices entirely the property that buyers' valuations do not need to be revealed, and blind-offer mechanisms are thus necessarily direct-revelation mechanisms. Hence, these mechanisms are not simple and suffer the same issue as to practicality, say, as articulated in Sandholm and Gilpin (2004). Still, they serve the purpose of proving an approximation ratio for enhanced sequential postedprice mechanisms with $d$-dimensionally dependent distributions. The proof of this result consists of three key ingredients:

- An upper bound on the optimal ex-post IC, ex-post IR revenue in terms of the solution of a linear program. This upper bound has the form of a relatively simple expression that is important for the definition and analysis of a blind offer mechanism that we define subsequently. This part of the proof generalizes a linear programming characterization introduced by Gupta and Nagarajan (2013) for the independent distribution setting.

- A proof for the fact that blind-offer mechanisms are powerful enough to extract a constant fraction of the optimal revenue of any instance. This makes crucial use of the linear program mentioned above.

- A conversion lemma showing that blind-offer mechanisms can be turned into enhanced sequential posted-price mechanisms while maintaining a fraction $\Omega(1 / d)$ of the revenue of the blind-offer mechanism. ${ }^{2}$

We note that the constant approximation ratio achievable by blind-offer mechanisms is mainly a means to an end, i.e., that of establishing an approximation for enhanced sequential postedprice mechanisms. Still, it may be of some interest to observe that this mechanism is an incentivecompatible mechanism that allocates items to buyers online (i.e., regardless of the order in which buyers are served) by means of threshold bids that are restricted to depend only on a buyer's conditional marginal distribution as defined in Section 2. Especially the latter property is important, as it does not allow for the type of pricing schemes used for approximations established in previous mechanisms (e.g., Ronen (2001), Dobzinski et al. (2011), and Chawla et al. (2014)), where the threshold prices rely in a stronger sense on the submitted bids. On the other hand, the Ronen (2001), Dobzinski et al. (2011), and Chawla et al. (2014) lookahead mechanisms achieve a much better approximation and apply to any matroid constraint.

While our focus is on proving the (non)existence of simple mechanisms that perform well in terms of revenue, we note the following about the computational complexity of our mechanisms:

\footnotetext{
${ }^{2}$ The sequential posted-price mechanism constructed in this lemma depends on a parameter $q$ that can be adjusted to trade off the amount of valuation elicitation against the hidden constant in the $\Omega(1 / d)$ expression.
} 
all the mechanisms that we use in our positive results run in polynomial time when the valuation distribution is given as a description of the valuation vectors together with their probability mass. ${ }^{3}$

Additionally, we note that all of our negative results hold for randomized mechanisms. On the other hand, our positive results only require randomization in a limited way. For our positive results for classical sequential posted-price mechanisms, only the offered prices need to be randomly chosen, while the order in which the buyers are picked is arbitrary. This makes these positive results hold for the order-oblivious (i.e., online) setting in which the mechanism has no control over the agent that is picked in each iteration. Our positive result for blind-offer mechanisms only requires randomized pricing in case $k<n$ and works for any ordering in which the buyers are picked, as long as the mechanism knows the ordering in advance. For $k=n$, randomized pricing is not needed. Our positive result for enhanced sequential posted-price mechanisms requires randomized pricing and the assumption that the mechanism can pick a uniformly random ordering of the buyers (i.e., it holds in the random order model (ROM) of arrivals).

Some definitions and proofs have been omitted in the main body of the article and can be found in the appendices.

\section{PRELIMINARIES AND NOTATION}

For $a \in \mathbb{N}$, we write $[a]$ to denote the set $\{1, \ldots, a\}$. We write $1[X]$ to denote the indicator function for property $X$ (i.e., it evaluates to 1 if $X$ holds, and to 0 otherwise). When $\vec{v}$ is a vector and $a$ is an arbitrary element, we denote by $\left(a, \vec{v}_{-i}\right)$ the vector obtained by replacing $v_{i}$ with $a$.

We face a setting where an auctioneer provides a service to $n$ buyers and is able to serve at most $k$ of the buyers. As mentioned in the introduction, the buyers have valuations for the service offered, which are drawn from a valuation distribution, defined as follows.

Definition 2.1 (Valuation Distribution). A valuation distribution $\pi$ for $n$ buyers is a probability distribution on $\mathbb{R}_{\geq 0}^{n}$.

We will assume throughout this article that $\pi$ is discrete, except for in Theorem 3.2, where we assume that various standard assumptions about continuous valuation distributions hold, such as regularity, the monotone hazard rate (MHR) condition, and affiliation. We refer the interested reader to Appendix A for definitions and a brief discussion of these properties.

We will use the following notation for conditional and marginal probability distributions. For an arbitrary probability distribution $\pi$, denote by $\operatorname{supp}(\pi)$ the support of $\pi$. Let $\pi$ be a discrete finite probability distribution on $\mathbb{R}^{n}$, and let $i \in[n], S \subset[n]$, and $\vec{v} \in \mathbb{R}^{n}$. We denote by $\vec{v}_{S}$ the vector obtained by removing from $\vec{v}$ the coordinates in $[n] \backslash S$. We denote by $\pi_{S}$ the probability distribution induced by drawing a vector from $\pi$ and removing the coordinates corresponding to index set $[n] \backslash S$. If $S=\{i\}$ is a singleton, we write $\pi_{i}$ instead of $\pi_{\{i\}}$, and if $S$ consists of all but one buyer $i$, we write $\pi_{-i}$ instead of $\pi_{[n] \backslash\{i\}}$. We denote by $\pi_{\vec{v}_{S}}$ the probability distribution of $\pi$ conditioned on the event that $\vec{v}_{S}$ is the vector of values on the coordinates corresponding to index set $S$. We denote by $\pi_{i, \vec{v}_{S}}$ the conditional marginal distribution of the coordinate of $\pi_{\vec{v}_{S}}$ that corresponds to buyer $i$. Again, in these cases, in the subscript we will also write $i$ instead of $\{i\}$ and $-i$ instead of $[n] \backslash\{i\}$. In particular, the conditional marginal distribution $\pi_{i, \vec{b}_{-i}}$ (i.e., the conditional marginal distribution for buyer $i$ when the other buyers bid $\vec{b}_{-i}$ ) will be central to our definition of blind-offer mechanisms.

\footnotetext{
${ }^{3}$ When the valuation distribution is not accessible in such a form and can instead only be sampled from, then standard sampling techniques can be used in order to obtain an estimate of the distribution. When this estimate is reasonably accurate (which should be the case when the distribution in question does not have extreme outliers), then the mechanisms in our article can still be used with only a small additional loss in revenue.
} 
Each of the buyers is interested in receiving the service at most once. The auctioneer runs a mechanism with which the buyers interact. In general, a mechanism consists of a specification of (1) the strategies available to the buyers and (2) a function that maps each vector of strategies chosen by the buyers to an outcome. The mechanism, when provided with a strategy profile of the buyers, outputs an outcome that consists of a vector $\vec{x}=\left(x_{1}, \ldots, x_{n}\right)$ and a vector $\vec{p}=\left(p_{1}, \ldots, p_{n}\right)$ : vector $\vec{x}$ is the allocation vector, i.e., the $(0,1)$-vector that indicates to which of the buyers the auctioneer allocates the service, and $\vec{p}=\left(p_{1}, \ldots, p_{n}\right)$ is the vector of prices that the auctioneer asks from the buyers. For outcome $(\vec{x}, \vec{p})$, the utility of a buyer $i \in[n]$ is $x_{i} v_{i}-p_{i}$. The auctioneer is interested in maximizing the revenue $\sum_{i \in[n]} p_{i}$ and is assumed to have full knowledge of the valuation distribution, but not of the actual valuations of the buyers.

We formalize the above as follows.

Definition 2.2. An instance is a triple $(n, \pi, k)$, where $n$ is the number of participating buyers, $\pi$ is the valuation distribution, and $k \in \mathbb{N}_{\geq 1}$ is the number of services that the auctioneer may allocate to the buyers. A deterministic mechanism $f$ is a function from $\times_{i \in[n]} \Sigma_{i}$ to $\{0,1\}^{n} \times \mathbb{R}_{\geq 0}^{n}$, for any choice of strategy sets $\Sigma_{i}, i \in[n]$. When $\Sigma_{i}=\operatorname{supp}\left(\pi_{i}\right)$ for all $i \in[n]$, mechanism $f$ is called a deterministic direct-revelation mechanism. A randomized mechanism $M$ is a probability distribution over deterministic mechanisms. For $i \in[n]$ and $\vec{s} \in \times_{j \in[n]} \sum_{j}$, we will denote $i$ 's expected allocation $\mathrm{E}_{f \sim M}\left[f(\vec{s})_{i}\right]$ by $x_{i}(\vec{s})$ and $i$ 's expected payment $\mathbf{E}_{f \sim M}\left[f(\vec{s})_{n+i}\right]$ by $p_{i}(\vec{s})$. (Whenever we use this notation, the mechanism $M$ will always be clear from context.)

Definition 2.3. Let $(n, \pi, k)$ be an instance and $M$ be a randomized direct-revelation mechanism for $(n, \pi, k)$. Mechanism $M$ is dominant strategy incentive compatible (dominant strategy IC) if and only if for all $i \in[n]$ and $\vec{s} \in \times_{j \in[n]} \operatorname{supp}\left(\pi_{j}\right)$ and $\vec{v}_{i} \in \operatorname{supp}\left(\pi_{i}\right)$,

$$
x_{i}\left(v_{i}, \vec{s}_{-i}\right) v_{i}-p_{i}\left(v_{i}, \vec{s}_{-i}\right) \geq x_{i}(\vec{s}) v_{i}-p_{i}(\vec{s}) .
$$

Mechanism $M$ is ex-post individually rational (ex-post $I R$ ) if and only if for all $i \in[n]$ and $\vec{s} \in$ $\operatorname{supp}(\pi)$

$$
x_{i}(s) v_{i}-p_{i}(s) \geq 0 .
$$

For convenience, we usually will not treat a mechanism explicitly as a probability distribution over deterministic mechanisms, but rather as the result of a randomized procedure that interacts in some way with the buyers. In this case, we say that a mechanism is implemented by that procedure. Sequential posted-price mechanisms are the mechanisms that are implemented by a particular such procedure, defined as follows.

Definition 2.4. A sequential posted-price mechanism for an instance $(n, \pi, k)$ is any mechanism that is implementable by iteratively selecting a buyer $i \in[n]$ that has not been selected in a previous iteration and proposing a price $p_{i}$ for the service, which the buyer may accept or reject. If $i$ accepts, he or she gets the service and pays $p_{i}$, resulting in a utility of $v_{i}-p_{i}$ for $i$. If $i$ rejects, he or she pays nothing and does not get the service, resulting in a utility of 0 for $i$. Once the number of buyers that have accepted an offer equals $k$, the process terminates. Randomization is allowed in the ordering of the buyers and for the prices offered.

We will initially only be concerned with sequential posted-price mechanisms. Later in the article we define and study the two generalizations of sequential posted-price mechanisms that we mentioned in the introduction.

Note that each buyer in a sequential posted-price mechanism has an obvious dominant strategy: he or she will accept whenever the price offered to him or her does not exceed his or her valuation, and will reject otherwise. Also, a buyer always ends up with a nonnegative utility when participating in a sequential posted-price mechanism. Thus, by the revelation principle (see, e.g., Börgers 
(2015)), a sequential posted-price mechanism can be straightforwardly converted into a dominant strategy IC and ex-post IR direct-revelation mechanism that achieves the same expected revenue.

Our interest lies in analyzing the revenue performance of a sequential posted-price mechanisms. We do this by comparing the expected revenue of such mechanisms to the maximum expected revenue that can be obtained by a mechanism that satisfies dominant strategy IC and ex-post IR. Thus, for a given instance, let $O P T$ be the maximum expected revenue that can be attained by a dominant strategy IC, ex-post IR mechanism and let $R E V(C)$ be the maximum expected revenue achievable by some class of mechanisms $C$. Our goal throughout this article is to derive (when possible) instance-independent lower and upper bounds on the ratio $R E V(C) / O P T$, when $C$ is the class of sequential posted-price mechanisms or one of the generalizations mentioned.

A more general class of mechanisms is formed by the ex-post incentive compatible, ex-post individually rational mechanisms.

Definition 2.5. Let $(n, \pi, k)$ be an instance and $M$ be a randomized direct-revelation mechanism for $(n, \pi, k)$. Mechanism $M$ is ex-post incentive compatible (ex-post IC) if and only if for all $i \in[n]$, $s_{i} \in \operatorname{supp}\left(\pi_{i}\right)$, and $\vec{v} \in \operatorname{supp}(\pi)$,

$$
x_{i}(\vec{v}) v_{i}-p_{i}(\vec{v}) \geq x_{i}\left(s_{i}, \vec{v}_{-i}\right) v_{i}-p_{i}\left(s_{i}, \vec{v}_{-i}\right) .
$$

In other words, a mechanism is ex-post IC if it is always (i.e., for any valuation vector) a pure equilibrium for the buyers to report their valuation. The difference between the ex-post IC notion and the dominant strategy IC notion is that dominant strategy IC requires that bidding one's true valuation is the best strategy to adopt, even when other buyers do not do so, while ex-post IC only requires that bidding one's true valuation is the best strategy if other buyers do so as well. ${ }^{4}$

In this work, we sometimes compare the expected revenue of our (dominant strategy IC and ex-post IR) mechanisms to the maximum expected revenue of the more general class of ex-post IC, ex-post IR mechanisms. This strengthens our positive results. We refer the interested reader to Roughgarden and Talgam-Cohen (2013) for a further discussion of and comparison between various notions of incentive compatibility and individual rationality.

\section{SEQUENTIAL POSTED-PRICE MECHANISMS}

We are interested in designing a posted-price mechanism that, for any given $n$ and valuation distribution $\pi$, achieves an expected revenue that lies only a constant factor away from the optimal expected revenue that can be achieved by a dominant strategy IC, ex-post IR mechanism. In this section, we show that this is unfortunately impossible. In fact, we will show that the nonconstant approximation ratios established for the examples in this section are asymptotically optimal.

We next prove the following theorem.

Theorem 3.1. For all $n \in \mathbb{N}_{\geq 2}$ and all $q \in[0,1]$, there exists a valuation distribution $\pi$ such that for all $k \in[n]$, there does not exist a sequential posted-price mechanism for instance $(n, \pi, k)$ that extracts a $q$ fraction of the expected revenue of the optimal dominant strategy IC, ex-post IR mechanism.

Proof. We first consider the unit supply setting, i.e., instances of the form $(n, \pi, 1)$. As a first step, we show that a posted-price mechanism cannot achieve a constant-factor approximation of the expected optimal social welfare, defined as

$$
\mathrm{OSW}=\mathrm{E}_{\vec{v} \sim \pi}\left[\max \left\{v_{i}: i \in[n]\right\}\right] .
$$

\footnotetext{
${ }^{4}$ Note that some literature claims that in settings similar to the one we study, the notions of ex-post IC and dominant strategy IC coincide. This holds only if the support of all conditional marginal distributions of a buyer are all equal, i.e., if for all $i \in[n]$ and for all $\vec{v}, \vec{v}^{\prime} \in \operatorname{supp}(\pi)$, it holds that $\operatorname{supp}\left(\pi_{i, v_{-i}}\right)=\operatorname{supp}\left(\pi_{i, v_{-i}^{\prime}}\right)$. We do not make the latter assumption in the present article, and therefore ex-post IC and dominant strategy IC are two distinct concepts in our setting.
} 
Let OR be the optimal revenue that a dominant strategy IC and ex-post IR mechanism can achieve on $(n, \pi, k)$. (OR depends on the valuation distribution $\pi$, but we assume that the valuation distribution is given, and implicit from context.) It is clear that OSW is an upper bound to OR regardless of $\pi$, since a dominant strategy IC and ex-post IR mechanism will not charge (in expectation) any buyer a higher price than its expected valuation.

Fix $m \in \mathbb{N}_{\geq 1}$ arbitrarily, and consider the case where $n=1$ and the valuation $v_{1}$ of the single buyer is taken from $\{1 / a: a \in[m]\}$ distributed such that $\pi_{1}(1 / a)=1 / m$ for all $a \in[m]$. In this setting, a posted-price mechanism will offer the buyer a price $p$, which the buyer subsequently accepts if and only if $v_{1} \geq p$. After that, the mechanism terminates.

Note that OSW $=(1 / m) \sum_{a=1}^{m}(1 / a)$. The expected revenue of the mechanism is

$$
\mathrm{RM}=p \operatorname{Pr}_{v_{1} \sim \pi_{1}}\left[v_{1} \geq p\right]=p \frac{|\{a: 1 / a \geq p\}|}{m}=\frac{\left|\left\{a: 1 / a \geq 1 / p^{-1}\right\}\right|}{m p^{-1}}=\frac{p^{-1}}{m p^{-1}}=\frac{1}{m} .
$$

Therefore:

$$
\lim _{m \rightarrow \infty} \frac{\mathrm{RM}}{\mathrm{OSW}}=\lim _{m \rightarrow \infty} \frac{1}{\sum_{a \in[m]} 1 / a}=\lim _{m \rightarrow \infty} \frac{1}{H(m)}=0,
$$

where $H(m)$ denotes the $m$ th harmonic number. So, no posted-price mechanism can secure in expectation a revenue that lies a constant factor away from the expected optimal social welfare. (Because our analysis is for an instance with only one buyer, this inapproximability result also holds for instances with independent valuations.)

We extend the above example in a simple way to a setting where the expected revenue of the optimal dominant strategy IC, ex-post IR mechanism is equal to the expected optimal social welfare.

Fix $m \in \mathbb{N}_{\geq 1}$ and consider a setting with two buyers, where the type vector $\left(v_{1}, v_{2}\right)$ takes values in $\{(1 / a, 1 / a): a \in[m]\}$ according to the probability distribution where $\pi((1 / a, 1 / a))=1 / m$ for all $a \in[m]$. A mechanism that always gives buyer 1 the service and charges buyer 1 the bid of buyer 2 is clearly dominant strategy IC and also clearly achieves a revenue equal to the optimal social welfare.

In this two-buyer setting, the value OSW is again $(1 / m) \sum_{a=1}^{m}(1 / a)$. By symmetry, we may assume without loss of generality that a posted-price mechanism works by first proposing a price $p_{1}$ to buyer 1 , and then proposing a price $p_{2}$ to buyer 2 if buyer 1 rejected the offer. Using arguments similar to Equation (1), we derive that the revenue of this mechanism is

$$
\begin{aligned}
\mathrm{RM} & =p_{1} \operatorname{Pr}_{\left(v_{1}, v_{2}\right) \sim \pi}\left[v_{1} \geq p_{1}\right]+p_{2} \operatorname{Pr}_{\left(v_{1}, v_{2}\right) \sim \pi}\left[v_{1}<p_{1} \cap v_{2} \geq p_{2}\right] \\
& =\frac{1}{m}+p_{2} \operatorname{Pr}_{\left(v_{1}, v_{2}\right) \sim \pi}\left[v_{1}<p_{1} \cap v_{2} \geq p_{2}\right] \leq \frac{1}{m}+p_{2} \operatorname{Pr}_{\left(v_{1}, v_{2}\right) \sim \pi}\left[v_{2} \geq p_{2}\right]=\frac{2}{m} .
\end{aligned}
$$

Therefore:

$$
\lim _{m \rightarrow \infty} \frac{\mathrm{RM}}{\mathrm{OR}}=\lim _{m \rightarrow \infty} \frac{\mathrm{RM}}{\mathrm{OSW}} \leq \lim _{m \rightarrow \infty} \frac{2}{\sum_{a \in[m]} 1 / a}=0 .
$$

The above example establishes the nonexistence of a good sequential posted-price mechanism in the case where the service has to be provided to a single buyer. Suppose now that the service can be provided to two buyers (i.e., $k=2$ ), and each buyer gets the service at most once. Consider again two buyers whose values are drawn from the probability distribution $\pi$ as defined above. As above, by symmetry, we may assume that our posted-price mechanism first proposes price $p_{1}$ to buyer 1, and then proposes either price $p_{2}$ or $p_{2}^{\prime}$ to buyer 2: $p_{2}$ is proposed in case the offer was rejected by buyer 1 , and $p_{2}^{\prime}$ is proposed otherwise. The difference with the previous analysis for the unit supply case is that the mechanism proposes a price to buyer 2 regardless of whether buyer 1 accepted the offer or not. 
We derive

$$
\begin{aligned}
\mathrm{RM}= & p_{1} \operatorname{Pr}_{\left(v_{1}, v_{2}\right) \sim \pi}\left[v_{1} \geq p_{1} \cap v_{2}<p_{2}^{\prime}\right]+p_{2} \operatorname{Pr}_{\left(v_{1}, v_{2}\right) \sim \pi}\left[v_{1}<p_{1} \cap v_{2} \geq p_{2}\right] \\
& +\left(p_{1}+p_{2}^{\prime}\right) \operatorname{Pr}_{\left(v_{1}, v_{2}\right) \sim \pi}\left[v_{1} \geq p_{1} \cap v_{2} \geq p_{2}^{\prime}\right] \\
\leq & \frac{2}{m}+\left(p_{1}+p_{2}^{\prime}\right) \operatorname{Pr}_{\left(v_{1}, v_{2}\right) \sim \pi}\left[v_{1} \geq p_{1} \cap v_{2} \geq p_{2}^{\prime}\right] \\
\leq & \frac{2}{m}+2 \max \left\{p_{1}, p_{2}^{\prime}\right\} \operatorname{Pr}_{\left(v_{1}, v_{2}\right) \sim \pi}\left[v_{1} \geq \max \left\{p_{1}, p_{2}^{\prime}\right\}\right] \leq \frac{4}{m} .
\end{aligned}
$$

The optimal-incentive-compatible mechanism works by giving the service to both buyers while charging the bid of buyer 1 to buyer 2, and charging the bid of buyer 2 to buyer 1 . The resulting expected revenue is exactly the expected optimal social welfare: $\mathrm{OR}=\mathrm{OSW}=(1 / m) \sum_{a=1}^{m}(2 / a)$. We therefore obtain

$$
\lim _{m \rightarrow \infty} \frac{\mathrm{RM}}{\mathrm{OR}}=\lim _{m \rightarrow \infty} \frac{\mathrm{RM}}{\mathrm{OSW}} \leq \lim _{m \rightarrow \infty} \frac{4}{\sum_{a \in[m]} 2 / a}=0 .
$$

The above yields an impossibility result for 2-limited supply. By adding to this instance dummy buyers that always have valuation 0 , we obtain an impossibility result for $k$-limited supply, where $k \in \mathbb{N}$.

We prove that the above impossibility result holds also in the continuous case even if we assume that all of the following conditions simultaneously hold: the valuation distribution is supported everywhere, is entirely symmetric, satisfies regularity, satisfies the monotone hazard rate condition, and satisfies affiliation; all the induced marginal distributions have finite expectation; and all the conditional marginal distributions are nonzero everywhere. We remark that Roughgarden and Talgam-Cohen (2013) showed that when all these assumptions are simultaneously satisfied, the optimal ex-post IC and ex-post IR mechanism is the Myerson mechanism, which is the same mechanism that is optimal in the independent value setting. Thus, these conditions make the correlated setting very similar to the independent one with respect to revenue maximization. Yet, our following result shows that, whereas posted-price mechanisms can achieve a constant approximation revenue in the independent setting, this result does not extend to the correlated setting.

THEOREM 3.2. For all $q \in[0,1]$, there exists a valuation distribution $\pi$ with the properties that

(1) $\pi$ has support $[0,1]^{2}$;

(2) the expectation $\mathbf{E}_{\vec{v} \sim \pi}\left[v_{i}\right]$ is finite for any $i \in\{1,2\}$;

(3) $\pi$ is symmetric in all its arguments;

(4) $\pi$ is continuous and nowhere zero on $[0,1]^{2}$;

(5) the conditional marginal densities $\pi_{i \mid \vec{v}_{-i}}$ are nowhere zero for any $\vec{v}_{-i} \in[0,1]^{n-1}$ and any $i \in\{1,2\}$;

(6) $\pi$ has a monotone hazard rate and is regular; and

(7) $\pi$ satisfies affiliation,

such that there does not exist a sequential posted-price mechanism on instance $(2, \pi, k)$, for $k \in\{1,2\}$ that extracts a $q$ fraction of the expected revenue of the optimal dominant strategy IC, ex-post IR mechanism.

Proof. Consider $c>1$ and $m \geq(c-\log c) / 2$ and set $M=1+1 / m$. Let $V$ be a random variable whose value is drawn over the support $[1 / m, 1]$ according to the probability density function

$$
f_{V}(x)=\frac{1}{(m-1) x^{2}} \text {. }
$$


Let $N_{1}$ and $N_{2}$ be two random variables whose values are independently drawn over the support $[0, M-v]$ according to the conditional density function

$$
f_{N \mid V}(z \mid V=v)=\frac{c^{-z} \ln (c)}{Z(v)}
$$

with $c>1$ and $Z(v)=1-c^{-(M-v)}$. Finally, let $\pi$ be the probability density function of the pair $(X, Y)=\left(V+N_{1}-1 / m, V+N_{2}-1 / m\right)$.

Before proving that this value distribution satisfies Properties 1 through 7, we show that there is a $c^{*}$ such that no sequential posted-price mechanism extracts constant revenue on this instance when $c=c^{*}$. To this aim, let us fix $\epsilon>0$. Observe that $\lim _{c \rightarrow \infty} f_{N \mid V}(0 \mid V=v)=1$ and $\lim _{c \rightarrow \infty} f_{N \mid V}(z \mid V=v)=0$ for any $z>0$. Hence, $\lim _{c \rightarrow \infty}|X-Y|=\lim _{c \rightarrow \infty} \mid(V-1 / m)-(V-$ $1 / m) \mid=0$. Thus, there must be $c^{*}=c(\epsilon)$ large enough such that $|X-Y|<\epsilon$.

Let us consider first the case that the service can be offered to only one buyer. In this setting, the following is a dominant strategy IC and ex-post IR mechanism: it offers to buyer 1 the service at a price equivalent to the valuation of buyer 2 minus $\epsilon$. If $c=c^{*}$, then in expectation, this mechanism extracts as revenue all but at most $2 \epsilon$ of the social welfare. The expected optimal social welfare (and thus the optimal expected revenue) is

$$
\mathrm{OR}=\mathrm{OSW} \approx \mathrm{E}_{v \sim f_{V}}[v-1 / m]=\int_{1 / m}^{1} \frac{v-1 / m}{(m-1) v^{2}} d v=\frac{\ln (m)}{m-1}-\frac{1}{m} \geq \frac{\ln (m-1)}{m-1},
$$

where we use the " $\approx$ " notation to suppress the $\epsilon$ term. A posted-price mechanism will offer buyer 1 a price $p_{1} \geq 0$, which the buyer subsequently accepts if and only if $X \geq p_{1}$. After that, if buyer 1 rejects, the mechanism offers a price $p_{2}$ to buyer 2 . Thus, if $p_{1} \in[0,1-1 / m]$, then

$$
\begin{aligned}
p_{1} \operatorname{Pr}_{v \sim f_{V}}\left[X \geq p_{1}\right] & \approx p_{1} \operatorname{Pr}_{v \sim f_{V}}\left[v \geq p_{1}+1 / m\right] \\
& =p_{1} \int_{p_{1}+1 / m}^{1} \frac{1}{(m-1) v^{2}} d v=\frac{p_{1}}{m-1}\left(\frac{1}{p_{1}+1 / m}-1\right) \leq \frac{1-p_{1}}{m-1} \leq \frac{1}{m-1} .
\end{aligned}
$$

Moreover, if $p_{1} \geq 1-1 / m$, then $p_{1} \operatorname{Pr}_{v \sim f_{V}}\left[X \geq p_{1}\right]=0$. Hence,

$$
\mathrm{RM}=p_{1} \operatorname{Pr}_{v \sim f_{V}}\left[X \geq p_{1}\right]+p_{2} \operatorname{Pr}_{v \sim f_{V}}\left[X<p_{1} \cap Y \geq p_{2}\right] \leq \frac{1}{m-1}+p_{2} \operatorname{Pr}_{v \sim f_{V}}\left[Y \geq p_{2}\right] \leq \frac{2}{m-1} .
$$

Therefore:

$$
\lim _{m \rightarrow \infty} \frac{\mathrm{RM}}{\mathrm{OR}}=\lim _{m \rightarrow \infty} \frac{\mathrm{RM}}{\mathrm{OSW}} \leq \lim _{m \rightarrow \infty} \frac{2}{\ln (m-1)}=0 .
$$

The case in which the service can be offered to both buyers is similar and omitted.

Let us now prove that, for every $c$, the distribution $\pi$ satisfies the desired properties. Properties 1 , 2, and 3 are trivial and can be immediately checked.

For $v \in[0,1]$, let $f_{X \mid V}(\cdot \mid V=v)$ and $f_{Y \mid V}(\cdot \mid V=v)$ be, respectively, the probability density functions of $X$ and $Y$ conditioned on the event that $V=v$. In order to establish the remaining properties, observe that $f_{X \mid V}(x \mid V=v)=f_{N \mid V}(x-v+1 / m \mid V=v)$ if $x+1 / m \geq v$ and 0 otherwise. Equivalently, $f_{Y \mid V}(y \mid V=v)=f_{N \mid V}(y-v+1 / m \mid V=v)$ if $y+1 / m \geq v$ and 0 otherwise. Consider now the triple $(X, Y, V)$. The joint density function of this triple is

$$
f_{X, Y, V}(x, y, v)=f_{X \mid Y, V}(x \mid Y=y, V=v) \cdot f_{Y \mid V}(y \mid V=v) \cdot f_{V}(v) .
$$

Note that $f_{X \mid Y, V}(x \mid Y=y, V=v)=f_{X \mid V}(x \mid V=v)$ if $\min \{x, y\}+1 / m \geq v$ and 0 otherwise. Then

$$
f_{X, Y, V}(x, y, v)=f_{N \mid V}(x-v+1 / m \mid V=v) \cdot f_{N \mid V}(y-v+1 / m \mid V=v) \cdot f_{V}(v)
$$


if $\min \{x, y\}+1 / m \geq v$ and 0 otherwise. Hence, we can compute $\pi$ as follows:

$$
\pi(x, y)=\int_{1 / m}^{1} f_{X, Y, V}(x, y, v) d v=\frac{\ln ^{2}(c)}{m-1} \cdot c^{-(x+y)} \cdot \int_{1 / m}^{\alpha+1 / m} \frac{c^{2 v}}{v^{2} Z(v)^{2}} d v
$$

where $\alpha=\min \{1-1 / m, x, y\}$. Note that the integrated function is continuous and positive in the interval in which it is integrated. Hence, the integral turns out to be nonzero. From this, we observe that $\pi(x, y)$ is continuous and nowhere zero on $[0,1]^{2}$, satisfying Property 4 .

Let us now derive the conditional probability density functions. By symmetry, it will be sufficient to focus only on $f_{X \mid Y}$ :

$$
\begin{aligned}
f_{X \mid Y}(x \mid Y=y) & =\int_{1 / m}^{1} f_{X \mid Y, V}(x \mid Y=y, V=v) \cdot f_{V}(v) d v=\frac{\ln (c)}{m-1} \cdot c^{-x} \cdot \int_{1 / m}^{\alpha+1 / m} \frac{c^{v}}{v^{2} Z(v)} d v \\
& =\frac{m^{2} c^{M} \ln (c)}{m-1} \cdot c^{-x} \cdot \int_{0}^{\alpha} \frac{1}{(m z+1)^{2}\left(c^{1-z}-1\right)} d z,
\end{aligned}
$$

where the last equality is obtained by setting $z=v-\frac{1}{m}$. It is now obvious that the conditional probability density functions are continuous and nowhere zero, as desired by Property 5 .

Let $\gamma(z)=1 /\left((m z+1)^{2}\left(c^{1-z}-1\right)\right), g(a)=\int_{0}^{a} \gamma(z) d z$ with $a \in[0,1]$, and let $\alpha^{\prime}=\min \{y, 1-$ $1 / m\}$. Then

$$
f_{X \mid Y}(x \mid Y=y)=\frac{m^{2} c^{M} \ln (c)}{m-1} \cdot c^{-x} \cdot \begin{cases}g(x), & \text { if } x<\alpha^{\prime} \\ g\left(\alpha^{\prime}\right), & \text { otherwise }\end{cases}
$$

Moreover, we have that

$$
\begin{aligned}
1-F_{X \mid Y}(x \mid Y=y) & =\int_{x}^{1} f_{X \mid Y}(z \mid Y=y) d z \\
& =\frac{m^{2} c^{M} \ln (c)}{m-1} \cdot \begin{cases}\int_{x}^{\alpha^{\prime}} c^{-z} g(z) d z+g\left(\alpha^{\prime}\right) \int_{\alpha^{\prime}}^{1} c^{-z} d z, & \text { if } x<\alpha^{\prime} ; \\
g\left(\alpha^{\prime}\right) \int_{x}^{1} c^{-z} d z=\frac{g\left(\alpha^{\prime}\right)\left(c^{-x}-c^{-1}\right)}{\ln (c)}, & \text { otherwise. }\end{cases}
\end{aligned}
$$

Hence, the inverse hazard rate is

$$
I(x)=\frac{1-F_{X \mid Y}(x \mid Y=y)}{f_{X \mid Y}(x \mid Y=y)}= \begin{cases}\frac{\int_{x}^{\alpha^{\prime}} c^{-z} g(z) d z+g\left(\alpha^{\prime}\right) \int_{\alpha^{\prime}}^{1} c^{-z} d z}{c^{-x} g(x)}, & \text { if } x<\alpha^{\prime} \\ \frac{1-c^{x-1}}{\ln (c)}, & \text { otherwise }\end{cases}
$$

We prove that $I(x)$ is nonincreasing in $x$ in the interval $[0,1]$ and thus $\pi$ has the monotone hazard rate and is, as a consequence, regular, as required by Property 6 .

Clearly, $I(x)$ is nonincreasing in $x$ in the interval $\left[\alpha^{\prime}, 1\right]$ since in this case $I(x)=\left(1-c^{x-1}\right) / \ln (c)$. Moreover, $I(x)$ does not have discontinuities for $x=\alpha^{\prime}$. So, it is sufficient to show that $I(x)$ is nonincreasing also in the interval $\left[0, \alpha^{\prime}\right]$. To this aim, observe that for $x<\alpha^{\prime}$,

$$
\begin{aligned}
\frac{d I(x)}{d x}= & \frac{d}{d x} \frac{\int_{x}^{\alpha^{\prime}} c^{-z} g(z) d z+g\left(\alpha^{\prime}\right) \int_{\alpha^{\prime}}^{1} c^{-z} d z}{c^{-x} g(x)} \\
= & \left(c^{-x} g(x) \frac{d}{d x} \int_{x}^{\alpha^{\prime}} c^{-z} g(z) d z-\int_{x}^{\alpha^{\prime}} c^{-z} g(z) d z \frac{d}{d x} c^{-x} g(x)\right. \\
& \left.+c^{-x} g(x) g\left(\alpha^{\prime}\right) \frac{d}{d x} \int_{\alpha^{\prime}}^{1} c^{-z} d z-g\left(\alpha^{\prime}\right) \int_{\alpha^{\prime}}^{1} c^{-z} d z \frac{d}{d x} c^{-x} g(x)\right) /\left(c^{-x} g(x)\right)^{2} .
\end{aligned}
$$


Observe that, according to the second fundamental theorem of calculus,

$$
\frac{d}{d x} \int_{x}^{\alpha^{\prime}} c^{-z} g(z) d z=-c^{-x} g(x)
$$

whereas

$$
\frac{d}{d x} c^{-x} g(x)=c^{-x} \gamma(x)-c^{-x} g(x) \ln (c),
$$

and $\frac{d}{d x} \int_{\alpha^{\prime}}^{1} c^{-z} d z=0$. Then,

$$
\frac{d I(x)}{d x}=-1+\left(\ln (c)-\frac{\gamma(x)}{g(x)}\right) \frac{\int_{x}^{\alpha^{\prime}} c^{-z} g(z) d z+g\left(\alpha^{\prime}\right) \int_{\alpha^{\prime}}^{1} c^{-z} d z}{c^{-x} g(x)}=-1+\left(\ln (c)-\frac{\gamma(x)}{g(x)}\right) I(x) .
$$

The result then follows by showing that $\gamma(x) / g(x) \geq \ln (c)$.

To this aim, let us consider the function $\gamma^{\prime}(z)=c^{z}\left(c^{1-z}-1\right)(m z+1)^{2}$ for $z \in[0, x]$. Note that

$$
\frac{d \gamma^{\prime}(z)}{d z}=2 m c(m z+1)\left(1-\frac{2 m+m z \log c+\log c}{c^{1-z}}\right) \leq 2 m c(m z+1)\left(1-\frac{2 m+\log c}{c}\right) \leq 0,
$$

where we used the fact that $m \geq(c-\log c) / 2$. Thus, $\gamma^{\prime}(z)$ is nonincreasing in its argument and, in particular,

$$
\gamma^{\prime}(z) \geq \gamma^{\prime}(x) \geq\left(c^{x}-1\right)\left(c^{1-x}-1\right)(m x+1)^{2} .
$$

By simple algebraic manipulation, it then follows that $\gamma(z) \leq \gamma(x) c^{z} /\left(c^{x}-1\right)$. Then

$$
\frac{\gamma(x)}{g(x)}=\frac{\gamma(x)}{\int_{0}^{x} \gamma(z) d z} \geq \frac{\gamma(x)}{\int_{0}^{x} \frac{c^{z}}{c^{x}-1} \gamma(x) d z}=\frac{c^{x}-1}{\int_{0}^{x} c^{z} d z}=\ln (c),
$$

as desired.

Set now $C=\ln ^{2}(c) /(m-1)$ and let

$$
h(a)=\int_{1 / m}^{\min \{1-1 / m, a\}+1 / m} \frac{c^{2 v}}{v^{2} Z(v)^{2}} d v
$$

with $a \in[0,1]$. Note that the integrated function is positive for any $v \in[1 / m, 1]$. Hence, the integral increases as the size of the interval in which it is defined increases. In other words, the function $h(a)$ is nondecreasing in $a$.

Consider now two pairs $(x, y)$ and $\left(x^{\prime}, y^{\prime}\right)$. Moreover, let $\hat{x}=\max \left\{x, x^{\prime}\right\}$ and $\check{x}=\min \left\{x, x^{\prime}\right\}$ and, similarly, define $\hat{y}$ and $\check{y}$. We show that $\pi(x, y) \pi\left(x^{\prime}, y^{\prime}\right) \leq \pi(\hat{x}, \hat{y}) \pi(\check{x}, \check{y})$, satisfying in this way also Property 7.

Indeed,

$$
\pi(x, y) \pi\left(x^{\prime}, y^{\prime}\right)=C^{2} c^{-\left(x+y+x^{\prime}+y^{\prime}\right)} h(\min \{x, y\}) h\left(\min \left\{x^{\prime}, y^{\prime}\right\}\right) .
$$

If $x \geq x^{\prime}$ and $y \geq y^{\prime}\left(x<x^{\prime}\right.$ and $y<y^{\prime}$, respectively), then $(\hat{x}, \hat{y})=(x, y)\left(\left(x^{\prime}, y^{\prime}\right)\right.$, respectively) and $(\check{x}, \check{y})=\left(x^{\prime}, y^{\prime}\right)((x, y)$, respectively), and the desired result immediately follows. Suppose instead that $(\hat{x}, \hat{y})=\left(x, y^{\prime}\right)$ and $(\check{x}, \check{y})=\left(x^{\prime}, y\right)$. Then

$$
\pi(\hat{x}, \hat{y}) \pi(\check{x}, \check{y})=C^{2} c^{-\left(x+y+x^{\prime}+y^{\prime}\right)} h\left(\min \left\{x, y^{\prime}\right\}\right) h\left(\min \left\{x^{\prime}, y\right\}\right) .
$$

We will prove that in this case, $h(\min \{x, y\}) h\left(\min \left\{x^{\prime}, y^{\prime}\right\}\right) \leq h\left(\min \left\{x, y^{\prime}\right\}\right) h\left(\min \left\{x^{\prime}, y\right\}\right)$. First, observe that on both sides one of the two factors must be $h\left(\min \left\{x, y, x^{\prime}, y^{\prime}\right\}\right)=h\left(\min \left\{x^{\prime}, y\right\}\right)$. Suppose without loss of generality that $\min \left\{x^{\prime}, y\right\}=y$. Then it is sufficient to prove that $h\left(\min \left\{x^{\prime}, y^{\prime}\right\}\right) \leq$ $h\left(\min \left\{x, y^{\prime}\right\}\right)$, or, since $h$ is nondecreasing, that $\min \left\{x^{\prime}, y^{\prime}\right\} \leq \min \left\{x, y^{\prime}\right\}$. If $x \leq y^{\prime}$, then $x^{\prime} \leq x \leq y^{\prime}$ by hypothesis and the claim follows. If $y^{\prime}<x$, then it immediately follows that $\min \left\{x^{\prime}, y^{\prime}\right\} \leq y^{\prime}$. The case that $(\hat{x}, \hat{y})=\left(x^{\prime}, y\right)$ and $(\check{x}, \check{y})=\left(x, y^{\prime}\right)$ is similar and hence omitted. 
We note that the above theorem can be easily extended to an arbitrary number of buyers, by adding dummy buyers whose valuation is independently drawn over the support $[0,1]$ according to the probability density function

$$
\pi(x)=\frac{c^{-x} \ln c}{1-c^{-1}} .
$$

Note that, with this extension, the resulting distribution $\pi$ does not satisfy the symmetry condition anymore, but on the other hand, the symmetry property is not necessary for the optimality of the Myerson mechanism to hold (see Roughgarden and Talgam-Cohen (2013)).

In the above theorems, we have demonstrated that it is impossible to have a sequential postedprice mechanism extract a constant fraction of the optimal revenue. More precisely, in our example instances, it was the case that the expected revenue extracted by every posted-price mechanism was a $\Theta(1 / \log (r))$ fraction of the optimal expected revenue, where $r$ is the ratio between the highest valuation and the lowest valuation in the support of the valuation distribution. A natural question that arises is whether this is the worst possible instance in terms of revenue extracted, as a function of $r$. Lavi and Nisan (2004) show that this is indeed the case. Indeed, they show an online randomized mechanism (and thus a sequential posted-price one) that achieves at least a $\Theta(1 / \log (r))$ fraction of the optimal revenue (see also the related paper of El-Yaniv et al. (2001)). Hence, we have the following proposition.

Proposition 3.3. Let $n \in \mathbb{N}_{\geq 1}$, and let $\pi$ be a probability distribution on $\mathbb{R}^{n}$. For every $k \in[n]$ there exists a sequential posted-price mechanism that, when run on instance $(n, \pi, k)$, extracts in expectation at least an $\Omega\left(1 / \log \left(r_{\pi}\right)\right)$ fraction of the expected revenue of the optimal dominant strategy IC and ex-post IR auction, where $r_{\pi}$ is the ratio between the highest and lowest valuation in the support of $\pi$.

We note that in many realistic scenarios, we do not expect the extremal valuations of the buyers to lie too far from each other, because often the valuation of a buyer is strongly impacted by prior objective knowledge of the value of the service to be auctioned. In Appendix B, we prove that the $\Omega\left(1 / \log \left(r_{\pi}\right)\right)$ bound can often be improved by adopting similar online mechanisms.

\section{ENHANCED SEQUENTIAL POSTED-PRICE MECHANISMS}

Our negative results on sequential posted-price mechanisms suggest that it is necessary for a mechanism to have means to retrieve the valuations of some of the buyers in order to improve the revenue performance. We accordingly propose a generalization of sequential posted-price mechanisms that possess this ability.

Definition 4.1. An enhanced sequential posted-price mechanism for an instance $(n, \pi, k)$ is a mechanism that can be implemented by iteratively selecting a buyer $i \in[n]$ that has not been selected in a previous iteration and performing exactly one of the following actions for buyer $i$ :

- Offer the service at price $p_{i}$ to buyer $i$, which the buyer may accept or reject. If $i$ accepts, he or she gets the service and pays $p_{i}$, resulting in a utility of $v_{i}-p_{i}$ for $i$. If $i$ rejects, he or she pays nothing and does not get the service, resulting in a utility of 0 for $i$.

- Ask $i$ for his or her valuation (in which case the buyer pays nothing and does not get the service).

Randomization is allowed.

This generalization is still dominant strategy IC and ex-post IR (i.e., the revelation principle allows us to convert them to dominant strategy IC, ex-post IR direct-revelation mechanisms). Actually, one can see that this mechanism still enjoys the stronger property of being obviously strategy-proof (Li 2015). Indeed, for enhanced sequential posted-price mechanisms, when a buyer 
gets asked his or her valuation, he or she has no incentive to lie, because in this case he or she does not get the service and pays nothing. ${ }^{5}$

Next, we analyze the revenue performance of enhanced sequential posted-price mechanisms. For this class of mechanisms, we prove that it is unfortunately still the case that no constant fraction of the optimal revenue can be extracted: the next section establishes an $O(1 / n)$ bound for enhanced sequential posted-price mechanisms. Enhanced sequential posted-price mechanisms nonetheless turn out to be more powerful than standard sequential posted-price mechanisms. Contrary to what we had for the former ones, enhanced mechanisms can be shown to extract a fraction of the optimal revenue that is independent of the valuation distribution. The $O(1 / n)$ bound turns out to be asymptotically tight. Our main positive result for enhanced sequential posted-price mechanisms is that when dependence of the valuation among the buyers is limited, then a constant fraction of the optimal revenue can be extracted. Specifically, in Section 4.2, we define the concept of $d$-dimensional dependence and prove that for an instance $(n, \pi, k)$ where $\pi$ is $d$-dimensionally dependent, there exists an enhanced sequential posted-price mechanism that extracts an $\Omega(1 / d)$ fraction of the optimal revenue. (This implies the claimed $\Omega(1 / n)$ bound by taking $d=(n-1)$.)

\subsection{Limitations of Enhanced Sequential Posted-Price Mechanisms}

Here we show that enhanced sequential posted-price mechanisms cannot extract a constant fraction of the expected revenue of the optimal dominant strategy IC, ex-post IR mechanisms. This is done by constructing a family of instances on which no enhanced sequential posted-price mechanism can perform well.

Theorem 4.2. For all $n \in \mathbb{N}_{\geq 2}$, there exists a valuation distribution $\pi$ such that for all $k \in[n]$, there does not exist an enhanced sequential posted-price mechanism for instance $(n, \pi, k)$ that extracts more than a $O(1 / n)$ fraction of the expected revenue of the optimal dominant strategy IC, ex-post IR mechanism.

Proof. We prove this for the case of $k=n$. The proof is easy to adapt for different $k$.

Let $n \in \mathbb{N}$ and $m=2^{n}$. We prove this claim by specifying an instance $I_{n}$ with $n$ buyers and proving that $\mathrm{RM}\left(I_{n}\right) / \mathrm{OR}\left(I_{n}\right) \in O(1 / n)$, where $\mathrm{RM}\left(I_{n}\right)$ denotes the largest expected revenue achievable by any enhanced sequential posted-price mechanism on $I_{n}$, and $\mathrm{OR}\left(I_{n}\right)$ denotes the largest expected revenue achievable by a dominant strategy IC, ex-post IR mechanism.

$I_{n}$ is defined as follows. Let $\epsilon \in \mathbb{R}_{>0}$ be a number smaller than $1 / \mathrm{nm}^{2}$. The valuation distribution $\pi$ is the one induced by the following process: (1) draw a buyer $i^{*}$ from the set $[n]$ uniformly at random; (2) draw numbers $\left\{c_{j}: j \in[n] \backslash\left\{i^{*}\right\}\right\}$ independently from $[m]$ uniformly at random; (3) for all $j \in[n] \backslash\left\{i^{*}\right\}$, set $v_{j}=c_{j} \epsilon$; and (4) set

$$
v_{i^{*}}=\frac{1}{\left(\sum_{j \in[n] \backslash\left\{i^{*}\right\}} c_{j}\right)_{\bmod m}+1} .
$$

Observe that for this distribution, it holds that for all $i \in[n]$, the valuation $v_{i}$ is uniquely determined by the valuations $\left(v_{j}\right)_{j \in[n] \backslash\{i\}}$. The optimal (direct-revelation) mechanism can therefore extract the total optimal social welfare as its revenue, as follows: it provides service to every buyer and sets the prices as follows. Let $b_{i}$ be the bid, i.e., the reported valuation of buyer $i$. Then,

\footnotetext{
${ }^{5} \mathrm{~A}$ problematic aspect from a practical point of view is that while there is no incentive for a buyer to lie, there is also no incentive to cooperate or to tell the truth. This problem is addressed in Appendix C.
} 
- if $b_{j}<1 / m$ for all $j \in[n] \backslash\{i\}$, charge $i$ a price of

$$
\frac{1}{\left(\sum_{j \in[n] \backslash\{i\}} b_{j} / \epsilon\right)_{\bmod m}+1} ;
$$

- otherwise, if there is a buyer $j \in[n] \backslash\{i\}$ and a number $c_{i} \in[m]$ such that

$$
b_{j}=\frac{1}{\left(c_{i}+\sum_{\ell \in[n] \backslash\{i, j\}} b_{\ell} / \epsilon\right)_{\bmod m}+1},
$$

then charge $i$ the price $c_{i} \epsilon$;

- otherwise, the mechanism charges $i$ an arbitrary price.

This mechanism is dominant strategy IC because the mechanism's decision to provide service to a buyer does not depend on his or her bid, and the price that a buyer is charged is not dependent on his or her own bid. This mechanism is ex-post IR because bidding truthfully always gives the buyer a utility of 0 . This mechanism achieves a revenue equal to the optimal social welfare because (by definition of the pricing rule) the price that a buyer is charged is equal to the valuation of that buyer, if all buyers bid truthfully. Also, note that the situation corresponding to the third point in the above specification of the mechanism will not occur when the buyers bid truthfully, and is only included for the sake of completely specifying the mechanism.

We argue that

$$
\mathrm{OR}\left(I_{n}\right)=\mathrm{E}_{\vec{v} \sim \pi}\left[\sum_{i \in[n]} v_{i}\right]=\sum_{i \in[n]} \mathrm{E}_{\vec{v} \sim \pi}\left[v_{i}\right]=\frac{(n-1) m \epsilon}{2}+\frac{H_{m}}{m},
$$

where the last equality follows because the expected valuation of each of the buyers is $((n-$ $1) / n)(m \epsilon / 2)+(1 / n)\left(H_{m} / m\right)$. This in turn holds because a buyer is elected as buyer $i^{*}$ with probability $1 / n$, and buyer $i^{*}$ 's marginal distribution is the distribution $\pi^{\prime}$ induced by drawing a value from the set $\{1 / a: a \in[m]\}$ uniformly at random. The latter distribution has already been encountered in the beginning of Section 3, where we concluded that its expected value is $H_{m} / \mathrm{m}$.

We now proceed to prove an upper bound on $\operatorname{RM}\left(I_{n}\right)$. Let $M$ be an arbitrary enhanced postedprice mechanism. Because $M$ is randomized, running $M$ on $I_{n}$ can be viewed as a probability distribution on a sample space of deterministic enhanced posted-price mechanisms that are run on $I_{n}$. We analyze the revenue of the mechanism conditioned on three disjoint events that form a partition of this sample space. Consider first the event $E_{1}$ that buyer $i^{*}$ gets asked for his or her valuation (when running $M$ on $I_{n}$ ). Conditioned on this event, the mechanism does not attain a revenue of more than $(n-1) m \epsilon$ because the revenue of each buyer in $[n] \backslash\left\{i^{*}\right\}$ is at most $m \epsilon$.

Consider next the event $E_{2}$ where buyer $i^{*}$ does not get asked for his or her valuation and buyer $i^{*}$ is not the last buyer that is selected. Then a price $p_{i^{*}}$ is proposed to $i^{*}$. Without loss of generality, $M$ draws $p_{i^{*}}$ from a probability distribution $P_{i^{*}}$ with finite support, and the choice of distribution $P_{i^{*}}$ depends on the sequence $S$ of buyers queried prior to $i^{*}$ together with the responses of the buyers in $S$. These responses take the form of a reported valuation in case a buyer in $S$ is asked to report his or her valuation, and the form of an accept/reject decision otherwise. Because $i^{*}$ is not the last buyer selected, $[n] \backslash\left(S \cup\left\{i^{*}\right\}\right)$ is nonempty, and there exists a buyer $j \in[n] \backslash\left(S \cup\left\{i^{*}\right\}\right)$ such that the choice of $P_{i^{*}}$ does not depend on $c_{j}$. By the fact that $c_{j}$ is drawn independently and uniformly at random from $[m]$ for all $j \in[n] \backslash\left(S \cup\left\{i^{*}\right\}\right)$ and by Equation (2), the marginal probability distribution of the valuation of buyer $i^{*}$ conditioned on $E_{2}$ is $\pi^{\prime}$ (which we defined above). Therefore,

$$
\mathbf{E}_{p_{i^{*}} \sim P_{i^{*}, \vec{v} \sim \pi}}\left[p_{i^{*}} \operatorname{Pr}\left[v_{i^{*}} \leq p_{i^{*}}\right]\right]=\mathbf{E}_{p_{i^{*} \sim P_{i^{*}}, \vec{v} \sim \pi^{\prime}}}\left[p_{i^{*}} \operatorname{Pr}\left[v_{i^{*}} \leq p_{i^{*}}\right]\right]=\frac{1}{m}
$$


where the last equality follows from Equation (1). Thus, the expected revenue of $M$ conditioned on $E_{2}$ is at most $1 / m+(n-1) m \epsilon$.

In the event $E_{3}$, the mechanism selects $i^{*}$ last. The expected revenue of $M$ conditioned on this event is at most the expected maximum social welfare: $(n-1) m \epsilon / 2+H_{m} / m$. The probability of event $E_{3}$ occurring is $1 / n$, because of the following. For $\ell \in[n]$, let $E_{3}^{\ell}$ be the event that $i^{*}$ is not the $\ell$ th buyer selected by $M$, and let $E_{3}^{<\ell}$ be the event that $i^{*}$ is not among the first $\ell-1$ buyers selected by $M$. Note that this means that $\operatorname{Pr}\left[E_{3}^{<1}\right]=1$. Then,

$$
\operatorname{Pr}\left[E_{3}\right]=\operatorname{Pr}\left[E_{3}^{<n}\right]=\operatorname{Pr}\left[E_{3}^{n-1} \mid E_{3}^{<n-1}\right] \operatorname{Pr}\left[E_{3}^{<n-1}\right]=\prod_{\ell \in[n-1]} \operatorname{Pr}\left[E_{3}^{\ell} \mid E_{3}^{<\ell}\right] .
$$

For every $\ell \in[n-1]$, and every set $S$ of $\ell-1$ buyers, it holds that if $i^{*} \notin S$ and $M$ selects $S$ as the first $\ell-1$ buyers, the probability of selecting buyer $i^{*}$ as the $\ell$ th buyer is $1 /(n-(\ell-1))$, by the definition of $\pi$ (particularly because buyer $i^{*}$ is a buyer picked uniformly at random). Therefore,

$$
\operatorname{Pr}\left[E_{3}\right]=\operatorname{Pr}\left[E_{3}^{<n}\right]=\prod_{\ell \in[n-1]}\left(1-\frac{1}{n-(\ell-1)}\right)=\prod_{\ell \in[n-1]} \frac{n-\ell}{n-\ell+1}=\frac{1}{n} .
$$

Thus, we obtain the following upper bound on $\operatorname{RM}\left(I_{n}\right)$ :

$$
\begin{aligned}
\operatorname{RM}\left(I_{n}\right) & \leq \operatorname{Pr}\left[E_{1}\right](n-1) m \epsilon+\operatorname{Pr}\left[E_{2}\right]\left(\frac{1}{m}+(n-1) m \epsilon\right)+\frac{1}{n}\left(\frac{(n-1) m \epsilon}{2}+\frac{H_{m}}{m}\right) \\
& \leq \frac{1}{m}+2(n-1) m \epsilon+\frac{m \epsilon}{2}+\frac{H_{m}}{m n} \leq 3(n-1) m \epsilon+\frac{H_{m}}{m n}+\frac{1}{m} .
\end{aligned}
$$

This leads us to conclude that

$$
\frac{\mathrm{RM}\left(I_{n}\right)}{\mathrm{OR}\left(I_{n}\right)} \leq \frac{3 n m \epsilon+H_{m} / m n+1 / m}{H_{m} / m}=\frac{3 n m^{2} \epsilon+H_{m} / n+1}{H_{m}} \leq \frac{H_{m} / n+4}{H_{m}}=\frac{1}{n}+\frac{4}{H_{2^{n}}} \in O\left(\frac{1}{n}\right) .
$$

\subsection{Revenue Guarantees for Enhanced Sequential Posted-Price Mechanisms}

In this section, we evaluate the revenue guarantees of the enhanced sequential posted-price mechanisms in the presence of a form of limited dependence that we will call $d$-dimensional dependence, for $d \in \mathbb{N}$. These are probability distributions for which it holds that the valuation distribution of a buyer conditioned on the valuations of the rest of the buyers can be retrieved by only looking at the valuations of a certain subset of $d$ buyers. Formally, we have the following definition.

Definition 4.3. A probability distribution $\pi$ on $\mathbb{R}^{n}$ is $d$-dimensionally dependent if and only if for all $i \in[n]$, there is a subset $S_{i} \subseteq[n] \backslash\{i\},\left|S_{i}\right|=d$ such that for all $\vec{v}_{-i} \in \operatorname{supp}\left(\pi_{-i}\right)$, it holds that $\pi_{i, \vec{v}_{S_{i}}}=\pi_{i, \vec{v}_{-i}}$.

Note that if $d=0$, then $\pi$ is a product of $n$ independent probability distributions on $\mathbb{R}$. On the other hand, the set of ( $n-1)$-dimensionally dependent probability distributions on $\mathbb{R}^{n}$ equals the set of all probability distributions on $\mathbb{R}^{n}$. This notion is useful in practice for settings where it is expected that a buyer's valuation distribution has a reasonably close relationship with the valuation of a few other buyers. As an example of one of these practical settings, consider the case where there is a "true" valuation $v$ for the item, an expert buyer that keeps this valuation, and remaining buyers who roughly follow the opinion of the expert buyer, so that their valuation is that of the expert buyer, influenced by independent noise. It is then sufficient to know the valuation of a single buyer, namely, the expert one, in order to retrieve the exact conditional distribution of any other buyer. Our definition of 1-dimensional dependence applies if such an expert buyer exists, even if the auctioneer does not know which buyer is the expert. Moreover, our definition 
of $d$-dimensional dependence is much more flexible than that: in the example just described, even if each bidder picks another bidder as his or her own "personal" expert and adds noise to the valuation of his or her expert, the distribution would remain 1-dimensionally dependent.

In general, $d$-dimensional dependence is relevant to many practical settings in which it is not necessary to have complete information about the valuations of all the other buyers in order to say something useful about the valuation of a particular buyer. This notion can be used to rule out the extreme kind of dependence used to prove Theorem 4.2: There, the distributions are not $(n-2)$-dimensionally dependent, because for each buyer $i$ it holds that the valuations of all buyers $[n] \backslash\{i\}$ are necessary in order to extract the valuation distribution of $i$ conditioned on the others' valuations.

It is important to note that the class of $d$-dimensional dependent distributions is a strict superset of the class of Markov random fields of degree $d$. A Markov random field of degree $d$ is a popular model to capture the notion of limited dependence, and for that model a more straightforward procedure than the one in the proof below exists for obtaining the same revenue guarantee, as we demonstrate in Appendix E. However, the notion of $d$-dimensional dependence is both more natural (for our setting) and much more general. In fact, we show in Appendix D that there exist distributions on $\mathbb{R}^{n}$ that are 1-dimensionally dependent, but are not a Markov random field of degree less than $n / 2$. In a sense, our definition of $d$-dimensional dependence resembles the limited dependence condition under which the Lovasz Local Lemma holds.

The main result that we will prove in this section is the following.

Theorem 4.4. For every instance $(n, \pi, k)$ where $\pi$ is $d$-dimensionally dependent, there exists an enhanced sequential posted-price mechanism of which the expected revenue is at least a $(2-$ $\sqrt{e}) /(16 d) \geq 1 /(46 d) \in \Omega(1 / d)$ fraction of the maximum expected revenue that can be extracted by an ex-post IC, ex-post IR mechanism. Moreover, if $k=n$, then there exists an enhanced posted-price mechanism of which the expected revenue is at least a $1 /(4 d)$ fraction of the maximum expected revenue that can be extracted by an ex-post IC, ex-post IR mechanism.

The proof of this theorem works by defining an appropriate enhanced sequential posted-price mechanism of which we prove that it satisfies the claimed revenue bound. We remark that this enhanced sequential posted-price mechanism requires a random order of arrival of buyers.

A corollary of this theorem is that the bound of Theorem 4.2 is asymptotically tight.

COROLlary 4.5. For every instance $(n, \pi, k)$, there exists an enhanced sequential posted-price mechanism of which the expected revenue is at least a $\Omega(1 / n)$ fraction of the maximum expected revenue that can be extracted by an ex-post IC, ex-post IR mechanism.

4.2.1 Blind-Offer Mechanisms. For proving our main result on enhanced sequential postedprice mechanisms (i.e., Theorem 4.4), we first introduce a more powerful class of mechanisms, which we call blind-offer mechanisms, and we prove that these can achieve a constant approximation of the optimal revenue. We finally show how we can convert blind-offer mechanisms into enhanced sequential posted-price mechanisms while losing only a factor of $1 / 4 d$ of the revenue, if the valuation distribution is $d$-dimensionally dependent.

In blind-offer mechanisms, all bidders submit a bid to the mechanism a priori, making them direct-revelation mechanisms. However, blind-offer mechanisms are still constrained to work by allocating the items through a sequence of threshold offers. The additional power of this class of mechanisms comes from the fact that the offer made to a buyer $i$ may now be determined to some extent by the submitted bids of all other buyers $[n] \backslash\{i\}$.

The following definition makes the notion of a blind-offer mechanism precise. 
Definition 4.6. Let $(n, \pi, k)$ be an instance and let $M$ be a direct-revelation mechanism for $(n, \pi, k)$. Mechanism $M$ is a blind-offer mechanism for $\pi$ if and only if it can be implemented as follows. Let $\vec{b}$ be the submitted bid vector and $(\vec{v})$ the vector of valuations drawn from the distribution:

(1) Terminate if $\vec{b} \notin \operatorname{supp}(\pi)$.

(2) Either terminate or select a buyer $i$ from the set of buyers that have not yet been selected, such that the choice of $i$ does not depend on $\vec{b}$.

(3) Offer buyer $i$ service at price $p_{i}$, where $p_{i}$ is drawn from a probability distribution that depends only on $\pi_{i, \vec{b}_{-i}}$ (hence, the distribution from which $p_{i}$ is drawn is determined by $\vec{b}_{-i}$ and it does not depend on $b_{i}$ ). Buyer $i$ accepts the offer and pays $p_{i}$ if and only if $v_{i} \leq p_{i}$

(4) Go to Step 2 if there is supply left, i.e., if the number of buyers who have accepted offers is less than $k$.

Randomization is allowed.

In the above definition, "offering a price" $p_{i}$ to buyer $i$ in the context of a direct-revelation mechanism means that we allocate the service to $i$ if $b_{i} \geq p_{i}$ and charge him or her a payment of $p_{i}$, and otherwise we do not allocate the service to $i$ and charge him or her nothing.

We would like to emphasize some important aspects of the definition of blind-offer mechanisms

- The offers made by a blind-offer mechanism are restricted in a subtle but crucial way: In point 3 of the above definition, the (possibly randomized) offer made to a buyer is determined entirely by the conditional marginal distribution $\pi_{i, \vec{b}_{-i}}$. The question whether or not a given direct-revelation mechanism is a blind-offer mechanism therefore depends (among other things) on the distribution $\pi$. To clarify this further, suppose that there exists a buyer $i$ and two distinct bid vectors $\vec{b}_{-i}, \vec{b}_{-i}^{\prime}$ of the other buyers such that $\pi_{i, \vec{b}_{-i}}=\pi_{i, \vec{b}_{-i}^{\prime}}$. In this case, the randomized offer made to buyer $i$ under bid vector $\vec{b}_{-i}$ is drawn from the same distribution as the randomized offer made to buyer $i$ under bid vector $\vec{b}_{-i}^{\prime}$. We will use the notion of blind-offer mechanisms in order to obtain an enhanced sequential posted-price mechanism with an $\Omega(1 / d)$ approximation factor, and the latter restriction is crucial in order to do so. We are not aware of any mechanism proposed in the previous literature that achieves a constant revenue approximation guarantee for arbitrarily correlated valuations and can be implemented as a blind-offer mechanism. However, without this approximation guarantee, it is easy to see that practically all dominant strategy IC, ex-post IR direct-revelation mechanisms with nonnegative payments are blind-offer mechanisms. This follows from well-known characterization results that state that each dominant strategy IC, ex-post IR mechanisms must work by means of threshold prices that are independent of a buyer's own bid. ${ }^{6}$ To illustrate the restrictiveness of blind-offer mechanisms further, in Appendix F, we give a simple example with independent valuations showing that a well-known mechanism called the GVCG-L mechanism (Chawla et al. 2014) is not a blind-offer mechanism. ${ }^{7}$

\footnotetext{
${ }^{6}$ This relates to what some readers may know as the taxation principle.

${ }^{7}$ In fact, instances with independently drawn valuations are an extreme case, since in such instances the marginal conditional distributions of the buyers are invariant. This implies that the prices offered by the mechanism are completely independent of the submitted bids for such instances, so that even the standard second price auction is not a blind-offer mechanism.
} 
- It is straightforward to see that (enhanced) sequential posted-price mechanisms are blindoffer mechanisms, when regarded as direct-revelation mechanisms.

- Despite that blind-offer mechanisms inherit from sequential posted-price mechanisms the property of making sequential threshold offers, they lose a lot of the conceptually attractive properties that sequential posted-price mechanisms possess. Mainly this is because blind-offer mechanisms are direct-revelation mechanisms. We emphasize that our motivation to define blind-offer mechanisms is merely to serve as a tool for obtaining an $\Omega(1 / d)$ approximate enhanced sequential posted-price mechanism. We do not consider blind-offer mechanisms to be attractive practical mechanisms in their own right.

We finally note that, for a quite subtle reason, blind-offer mechanisms are not inherently ex-post IC, and the constant-approximate blind-offer mechanism that we propose below is not incentive compatible either. However, as said above, our main aim is not to design an ex-post IC blind-offer mechanism. Rather, we aim to use blind-offer mechanisms to devise $\Omega(1 / d)$-approximate enhanced sequential posted-price mechanisms (which are ex-post IC by definition). In order to do so, we will see that it suffices to have merely a non-ex-post IC blind-offer mechanism that extracts a constant fraction of the optimal revenue. In Appendix G, we elaborate further on the reason why blind-offer mechanisms are not ex-post IC in general, and we show furthermore how we can nonetheless turn any blind-offer mechanism into a dominant strategy IC blind-offer mechanism. The latter implies that blind-offer mechanisms are actually able to approximate the optimal revenue within a constant factor. This could be of some theoretical interest, since a blind-offer mechanism sets threshold prices as a function of only the marginal conditional distributions of the buyers. As already noted, to the best of our knowledge, all revenue approximation mechanisms proposed in other literature use instead threshold prices that require a more refined dependence on the submitted bid vector (see Appendix F for an illustration of this fact for the case of the GVCG-L mechanism).

We will prove in Section 4.2.3 that blind-offer mechanisms can always extract a constant fraction of the optimal revenue, without making any assumptions on the valuation distribution. Specifically, we will prove the following theorem.

THEOREM 4.7. For every instance $(n, \pi, k)$, there is blind-offer mechanism for which the expected revenue is at least $a(2-\sqrt{e}) / 4 \approx 0.088$ fraction of the maximum expected revenue that can be extracted by an ex-post IC, ex-post IR mechanism. Moreover, if $k=n$, then there is a blind-offer mechanism for which the expected revenue equals the maximum expected revenue that can be extracted by an ex-post IC, ex-post IR mechanism.

4.2.2 The Conversion Lemma. Before proving Theorem 4.7, we show how to use blind-offer mechanisms in order to prove Theorem 4.4. This proof works by means of a conversion lemma that turns a blind-offer mechanism into an enhanced sequential posted-price mechanism. This conversion lemma works by preselecting a set of buyers whose valuations are extracted, so that the conditional marginal distribution of the other buyers is known with high (at least constant) probability. The prices offered to the other buyers are then made according to the pricing function of the blind-offer mechanism. Observe that, for this conversion lemma, it is vital that the pricing of blind-offer mechanisms is a function of only the conditional marginal distributions of the players. This also implies that no previously known mechanism, such as GVCG-L, can be used in place of the blind-offer mechanism.

Let us now explain how this conversion works. Let $M$ be any blind-offer mechanism and let $q \in[0,1]$. We construct from $M$ the following enhanced sequential posted-price mechanism $M_{q}$. Let $p_{i}^{M}(\vec{v})$ be the expected payment by buyer $i \in[n]$ under mechanism $M$ when $\vec{v}$ are the reported valuations. The expectation is with respect to the distribution $\pi$ as well as with respect to any 
randomization in the mechanism. Note that with payment, we intend the amount of money paid ex-post by a buyer, rather than the price offered by the mechanism to a buyer. For a buyer $i$, let $S_{i} \subseteq$ $[n] \backslash\{i\}$ be a set of $d$ buyers such that $\pi_{i, \vec{v}_{S_{i}}}=\pi_{i, \vec{v}_{-i}}$ for all $\vec{v} \in \mathbb{R}^{n}$. Mechanism $M_{q}$ first partitions $[n]$ into two sets $A$ and $B=[n] \backslash A$. It does so by placing each buyer independently with probability $q$ in set $A$, and placing him or her in set $B$ otherwise. Then, the mechanism retrieves the vector $\vec{v}_{A}$ by asking the buyers in $A$ for their valuations. The existence of $M$ implies the existence of a blindoffer mechanism $M_{B}$ that only makes offers to buyers in $B$ such that the expected price $p_{i}^{M_{B}}(\vec{v})$ that $M_{B}$ offers to a buyer in $B$ is at least $p_{i}^{M}(\vec{v})$ : This can be achieved by doing the same as $M$, but refraining from offering to buyers in $A .{ }^{8}$ Mechanism $M_{q}$ offers each buyer $i \in B$ a price that is determined by simulating $M_{B}$ as follows: offer the same prices as $M_{B}$ would to only the buyers $i \in B$ such that $S_{i} \subseteq A$, and skip all other buyers in $B$. (Note, then, that a buyer $i$ in $B$ is skipped if and only if there exists $\vec{v}_{-i} \in \operatorname{supp}\left(\pi_{-i}\right)$ agreeing with $\vec{v}_{A}$ such that $\pi_{i, \vec{v}_{-i}} \neq \pi_{i, \vec{v}_{A}}$.) The probability that any buyer is offered the price of $M_{B}$ is thus exactly $(1-q) q^{d}$, i.e., the probability that $i \in B$ and that $S_{i} \subseteq A$. The desired revenue guarantee of $M_{q}$ follows straightforwardly from this fact, and we formalize this in the following lemma.

LeMma 4.8. Let $\alpha \in[0,1]$ and let $(n, \pi, k)$ be an instance where $\pi$ is $d$-dimensionally dependent. If there exists a blind-offer mechanism $M$ for $(n, \pi, k)$ that extracts in expectation at least an $\alpha$ fraction of the expected revenue of the optimal dominant strategy IC, ex-post IR mechanism, then $M_{q}$ is an enhanced sequential posted-price mechanism that extracts in expectation at least an $\alpha / \max \{4 d, 1\}$ fraction of the expected revenue of the optimal ex-post IC, ex-post IR mechanism, where $q=1$ ifd $=1$ and $q=1-1 / d$ if $d>1$.

Proof. Let $P$ be the distribution (induced by mechanism $M_{q}$ ) on the set of partitions of $[n]$ into two sets. For $i \in[n]$, let $S_{i} \subseteq[n] \backslash\{i\}$ be the set of $d$ buyers such that $\pi_{i, \vec{v}_{S_{i}}}=\pi_{i, \vec{v}_{-i}}$ for all $\vec{v} \in \mathbb{R}^{n}$. For $T \subseteq[n]$, let $p_{i}(T, \vec{v})$ be the expected payment by buyer $i \in T$ under $M_{q}$, conditioned on the event that $B=T$ and $S_{i} \subseteq A$. Note that $p_{i}(T, \vec{v}) \geq p_{i}^{M_{B}}(\vec{v}) \geq p_{i}^{M}(\vec{v})$. Therefore, the expected revenue of $M_{q}$ is

$$
\begin{aligned}
\sum_{\vec{v} \in \operatorname{supp}(\pi)} \pi(\vec{v}) \sum_{i \in[n]} \operatorname{Pr}_{\{A, B\} \sim P}\left[i \in B \cap S_{i} \subseteq A\right] p_{i}(B, \vec{v}) & =\sum_{\vec{v} \in \operatorname{supp}(\pi)} \pi(\vec{v}) \sum_{i \in[n]}(1-q) q^{d} p_{i}(B, \vec{v}) \\
& \geq(1-q) q^{d} \sum_{\vec{v} \in \operatorname{supp}(\pi)} \pi(\vec{v}) \sum_{i \in[n]} p_{i}^{M}(\vec{v}) .
\end{aligned}
$$

The last (double) summation is at least $\alpha$ times the expected revenue of the optimal dominant strategy IC, ex-post IR mechanism, by definition of $M$. Therefore, this mechanism extracts at least a $(1-q) q^{d} \alpha$ fraction of the optimal revenue. For $d=0$, it is optimal to set $q=0$, which results in an enhanced sequential posted-price mechanism of which the revenue is $\alpha$-approximately optimal. For $d=1$, it is optimal to set $q=1 / 2$, which results in an $(\alpha / 4)$-approximately optimal enhanced sequential posted-price mechanism. For $d \geq 2$, setting $q=1-1 / d$ will achieve the desired approximation ratio, since $\lim _{d \rightarrow \infty}(1-1 / d)^{d}=1 / e$. Moreover, $(1-1 / d)^{d}$ is increasing in $d$, and equals $1 / 4$ for $d=2$.

Theorem 4.4 now follows directly by combining Theorem 4.7 and Lemma 4.8 above. We note that in the above proof, it is easy to see that we can decrease the fraction $q$ of buyers being probed for their valuation at the cost of worsening the approximation guarantee. For example, when $d \geq 3$, if we set $q=1-2 / d$, then $\lim _{d \rightarrow \infty}(1-2 / d)^{d}=(1 / e)^{2}$.

\footnotetext{
${ }^{8}$ The fact that buyers in $A$ are not made an offer might cause the supply to run out less quickly, and could increase the expected payment of a buyer in $B$.
} 
4.2.3 Revenue Guarantees for Blind-Offer Mechanisms. Let us now formally prove Theorem 4.7.

We need to establish some intermediate results in order to build up to a proof for the above theorem. First, we derive an upper bound on the revenue of the optimal ex-post IC, ex-post IR mechanism. For a given instance $(n, \pi, k)$, consider the linear program with variables $\left(y_{i}(\vec{v})\right)_{i \in[n], \vec{v} \in \operatorname{supp}(\pi)}$ where the objective is

$$
\max \sum_{i \in[n]} \sum_{\vec{v}_{-i} \in \operatorname{supp}\left(\pi_{-i}\right)} \pi_{-i}\left(\vec{v}_{-i}\right) \sum_{v_{i} \in \operatorname{supp}\left(\pi_{i, \vec{v}_{-i}}\right)} \operatorname{Pr}_{v_{i}^{\prime} \sim \pi_{i, \vec{v}_{-i}}}\left[v_{i}^{\prime} \geq v_{i}\right] v_{i} y_{i}\left(v_{i}, \vec{v}_{-i}\right)
$$

subject to the constraints

$$
\begin{aligned}
& \forall i \in[n], \vec{v}_{-i} \in \operatorname{supp}\left(\pi_{-i}\right): \sum_{v_{i} \in \operatorname{supp}\left(\pi_{i, \vec{v}_{-i}}\right)} y_{i}(\vec{v}) \leq 1, \\
& \forall \vec{v} \in \operatorname{supp}(\pi): \sum_{i \in[n]} \sum_{v_{i}^{\prime} \in \operatorname{supp}\left(\pi_{i, \vec{v}_{-i}}\right): v_{i}^{\prime} \leq v_{i}} y_{i}\left(v_{i}^{\prime}, \vec{v}_{-i}\right) \leq k, \\
& \forall i \in[n], \vec{v} \in \operatorname{supp}(\pi): y_{i}(\vec{v}) \geq 0 .
\end{aligned}
$$

The next lemma states that the solution to this linear program forms an upper bound on the revenue of the optimal mechanism, and that the solution to the above linear program is integral in case $k=n$.

Lemma 4.9. For any instance $(n, \pi, k)$, the linear program (Equations (3) to (6)) upper bounds the maximum expected revenue achievable by an ex-post IC, ex-post IR mechanism. Moreover, when $k=n$, the optimal solution to (Equations (3) to (6)) is to set $y_{i}\left(v_{i}, \vec{v}_{-i}\right)$ to 1 for the value $v_{i}$ that maximizes $v_{i} \operatorname{Pr}_{v_{i}^{\prime} \sim \pi_{i, v_{-i}}}\left[v_{i}^{\prime} \geq v_{i}\right]$ (for all $i \in[n], \vec{v} \in \operatorname{supp}(\pi)$ ).

Intuitively, it is clear that this lemma is true: in the linear program, the value $y_{i}\left(v_{i}, v_{-i}\right)$ can be interpreted as the probability of setting a threshold price equal to $v_{i}$ to buyer $i$, given that the valuations of the other buyers are $v_{-i}$. The linear program is therefore expressing that in the optimal ex-post IC, ex-post IR mechanism, some optimal random threshold price is offered to each of bidder $i$, which only depends on the valuations $v_{-i}$. Lastly, the constraint in Equation (5) expresses that if we make such offers independently to each buyer, then the supply should not be exhausted in expectation, which is clearly a prerequisite for any mechanism to be feasible. Characterizations of this flavor, i.e., in terms of threshold offers, are well known in mechanism and auction design, and similar linear-programming-based techniques have been used before as well (Dobzinski et al. 2011; Gupta and Nagarajan 2013). However, such results are not known for this exact setting with limited supply and arbitrary discrete valuation distributions. Due to the similarity to results that appeared previously, we defer the proof of Lemma 4.9 to Appendix H, but nonetheless we note that our proof differs in various subtle but important ways from those results published previously.

Using Lemma 4.9, we now prove our main result about blind-offer mechanisms. We first handle the case of unlimited supply, for which we use the following blind-offer mechanism, which is in fact equivalent to the GVCG-Lmechanism of Chawla et al. (2014).

Definition 4.10. Consider an instance $(n, \pi, n)$. For $i \in[n]$ and $\vec{v}_{-i} \in \operatorname{supp}\left(\pi_{-i}\right)$, fix $\hat{p}_{i, \vec{v}_{-i}}$ to be any value in the set $\arg _{p} \max \left\{p \operatorname{Pr}_{v_{i} \sim \pi_{i, \vec{v}_{i}}}\left[p \leq v_{i}\right]: p \in \mathbb{R}\right\}$. Define $M_{\pi}^{n}$ to be the following blindoffer mechanism for allocating service to $n$ buyers when the valuations of these buyers are drawn from $\pi$.

Let $\overrightarrow{\vec{b}}$ be the submitted vector of bids. For $i \in[n]$, if $\vec{b}_{-i} \in \operatorname{supp}\left(\pi_{-i}\right)$ and $b_{i} \geq \hat{p}_{i, \vec{b}_{-i}}$, then $M_{\pi}^{n}$ gives service to $i$ and charges $i$ the price $\hat{p}_{i, \vec{b}_{-i}}$. If $b_{i}<\hat{p}_{i, \vec{b}_{-i}}$, then the price charged to $i$ is 0 , and 
$i$ is not given service. Otherwise, if $\vec{b}_{-i} \notin \operatorname{supp}\left(\pi_{-i}\right)$, the price charged to $i$ is 0 and $i$ is not given service.

LEMma 4.11. For instance $(n, \pi, n)$, mechanism $M_{\pi}^{n}$ extracts the maximum revenue among the class of ex-post IC, ex-post IR mechanisms.

Proof. Denote by $p_{i}(\vec{v})$ the price charged to buyer $i \in[n]$ when the buyers have valuation vector $\vec{v} \in \operatorname{supp}(\pi)$. We can write the expected revenue of $M_{\pi}^{n}$ as follows:

$$
\begin{aligned}
\mathrm{E}_{\vec{v} \sim \pi}\left[\sum_{i \in[n]} p_{i}(\vec{v})\right] & =\sum_{i \in[n]} \mathrm{E}_{\vec{v} \sim \pi}\left[p_{i}(\vec{v})\right]=\sum_{i \in[n]} \sum_{\vec{v} \in \operatorname{supp}(\pi)} \pi(\vec{v}) p_{i}(\vec{v}) \\
& =\sum_{i \in[n]} \sum_{\vec{v}_{-i} \in \operatorname{supp}\left(\pi_{-i}\right)} \pi_{-i}\left(\vec{v}_{-i}\right) \operatorname{Pr}_{v_{i} \sim \pi_{i,} \vec{v}_{-i}}\left[v_{i} \geq \hat{p}_{i, \vec{v}_{-i}}\right] \hat{p}_{i, \vec{v}_{-i}}
\end{aligned}
$$

Lastly, by Lemma 4.9 and the objective function in Equation (3) of the linear program, we conclude that the latter expression is equal to the solution of the linear program, which is an upper bound on the optimal revenue among all ex-post IC, ex-post IR mechanisms by Theorem 4.9.

For the case of $k$-limited supply where $k<n$, things are somewhat more complicated. Indeed, there does not seem to exist a blind-offer mechanism as simple and as elegant as $M_{\pi}^{n}$. However, we are still able to define a blind-offer mechanism that extracts at least a constant fraction of the optimal revenue.

Definition 4.12. Let $(n, \pi, k)$ be an arbitrary instance. Let $\left(y_{i}^{*}(\vec{v})\right)_{i \in[n]}$ be the optimal solution to the linear program (Equations (3) to (6)) corresponding to this instance.

Let $M_{\pi}^{k}$ be the blind-offer mechanism that does the following: let $\vec{v}$ be the vector of submitted valuations. Iterate over the set of buyers such that in iteration $i$, buyer $i$ is picked. In iteration $i$, select one of the following options: offer service to buyer $i$ at a price $p$ for which it holds that $y_{i}^{*}\left(p, \vec{b}_{-i}\right)>0$, or skip buyer $i$. The probabilities with which these options are chosen are as follows: price $p$ is offered with probability $y_{i}^{*}\left(p, \vec{b}_{-i}\right) / 2$, and buyer $i$ is skipped with probability $1-\sum_{p^{\prime} \in \operatorname{supp}\left(\pi_{i, \vec{b}_{-i}}\right)} y_{i}^{*}\left(p, \vec{b}_{-i}\right) / 2$. The mechanism terminates once $k$ buyers have accepted an offer, or when iteration $n+1$ is reached.

LEMma 4.13. On instance $(n, \pi, k)$, the expected revenue of blind-offer mechanism $M_{\pi}^{k}$ is at least a $(2-\sqrt{e}) / 4 \approx 0.088$ fraction of the expected revenue of the optimal ex-post IC, ex-post IR mechanism.

Proof. We will show that the expected revenue of $M_{\pi}^{k}$ is at least

$$
\frac{2-\sqrt{e}}{4} \sum_{i \in[n]} \sum_{\vec{v}_{-i} \in \operatorname{supp}\left(\pi_{-i}\right)} \pi_{-i}\left(\vec{v}_{-i}\right) \sum_{v_{i} \in \operatorname{supp}\left(\pi_{i, \vec{v}_{-i}}\right)} \operatorname{Pr}_{v_{i}^{\prime} \sim \pi_{i, \vec{v}_{-i}}}\left[v_{i}^{\prime} \geq v_{i}\right] v_{i} y_{i}^{*}\left(v_{i}, \vec{v}_{-i}\right),
$$

which is by Theorem 4.9 and the objective function (Equation (3)) a $(2-\sqrt{e}) / 4$ fraction of the expected revenue of the optimal ex-post IC, ex-post IR mechanism.

For a vector of valuations $\vec{v} \in \operatorname{supp}(\pi)$ and a buyer $i \in[n]$, denote by $D_{i, \vec{v}_{-i}}$ the probability distribution from which mechanism $M_{\pi}^{k}(\vec{v})$ draws a price that is offered to buyer $i$, in case iteration 
$i \in[n]$ is reached (as described in Definition 4.12). We let $V$ be a number that exceeds $\max \left\{v_{i}: i \in\right.$ $[n], \vec{v} \in \operatorname{supp}(\pi)\}$ and represent by $V$ the option where $M_{\pi}^{k}(\vec{v})$ chooses the "do nothing"-option during iteration $i$, so that $D_{i, \vec{v}_{-i}}$ is a probability distribution on the set $\{V\} \cup\left\{v_{i}: y_{i}^{*}\left(v_{i}, \vec{v}_{-i}\right)>0\right\}$. Let us formulate an initial lower bound on the expected revenue of $M_{\pi}^{k}$ :

$$
\begin{aligned}
& \mathbf{E}_{\vec{v} \sim \pi}\left[\text { revenue of } M_{\pi}^{k}(\vec{v})\right] \\
& =\mathrm{E} \underset{\substack{\vec{v} \sim \pi, p_{1} \sim D_{1, \vec{v}_{-1}},}}{\sim}\left[\sum_{i \in[n]} p_{i} \mathbf{1}\left[p_{i} \leq v_{i}\right] 1\left[\left|\left\{j \in[i-1]: p_{j} \leq v_{j}\right\}\right|<k\right]\right] \\
& p_{n} \sim D_{n, \vec{v}_{-n}}
\end{aligned}
$$

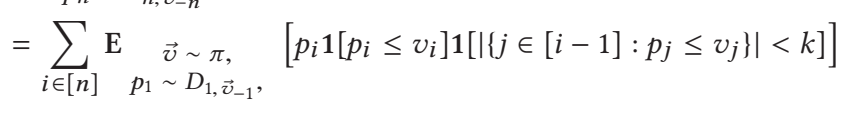

$$
\begin{aligned}
& \begin{array}{c}
\vdots \\
p_{n} \sim D_{n, \vec{v}_{-n}}
\end{array} \\
& =\sum_{i \in[n]} \sum_{\vec{v} \in \operatorname{supp}(\pi)} \pi(\vec{v}) \sum_{p_{1} \in \operatorname{supp}\left(D_{1, \vec{v}_{-1}}\right)} p_{i} 1\left[p_{i} \leq v_{i}\right] 1\left[\left|\left\{j \in[i-1]: p_{j} \leq v_{j}\right\}\right|<k\right] \prod_{j \in[i]} D_{j, \vec{v}_{-j}}\left(p_{j}\right) \\
& p_{i} \in \operatorname{supp}\left(D_{i, \vec{v}_{-i}}\right) \\
& =\sum_{i \in[n]} \sum_{\vec{v} \in \operatorname{supp}(\pi)} \pi(\vec{v}) \sum_{\substack{p_{i} \in \operatorname{supp}\left(D_{i, \vec{v}_{-i}}\right) \\
: p_{i} \leq v_{i}}} p_{i} D_{i, \vec{v}_{-i}}\left(p_{i}\right) \sum_{\substack{p_{1} \in \operatorname{supp}\left(D_{1, \vec{v}_{-1}}\right) \\
:}} \prod_{j \in[i-1]} D_{j, \vec{v}_{-j}}\left(p_{j}\right) \\
& p_{i-1} \in \operatorname{supp}\left(D_{\left.i-1, \vec{v}_{-(i-1)}\right)}\right. \\
& :\left|\left\{j \in[i-1] \mid p_{j} \leq v_{j}\right\}\right|<k
\end{aligned}
$$

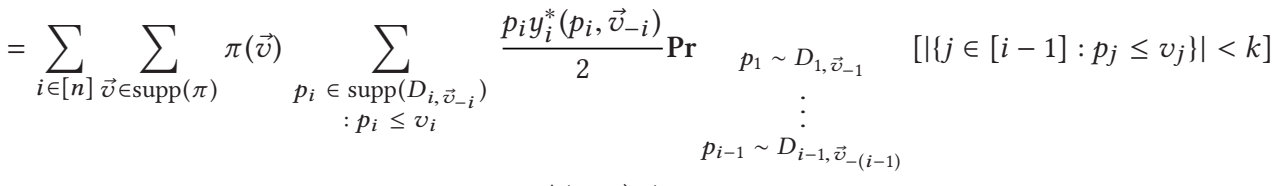

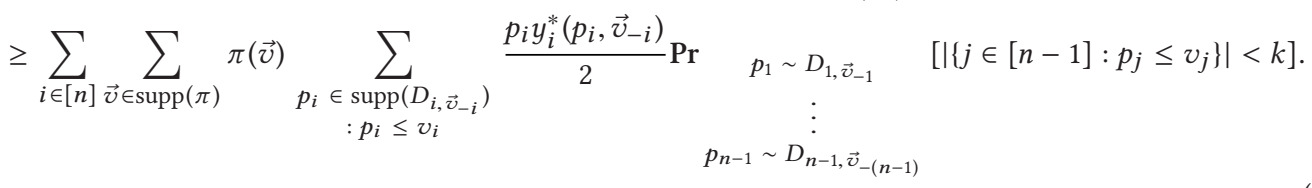

For the second equality, we applied linearity of expectation; the third equality follows from the definition of expected value; to obtain the fourth equality, we eliminate the indicator functions by removing the appropriate terms from the summation; in the fifth equality, we substitute $D_{i, \vec{v}_{-i}}\left(p_{i}, \vec{v}_{-i}\right)$ and $D_{j, \vec{v}_{-j}}\left(p_{j}^{\prime}, \vec{v}_{-j}\right)$ by concrete probabilities. For the last inequality, we lower bounded the last probability in the expression by replacing $i$ by $n$.

For $\vec{v} \in \operatorname{supp}(\pi)$ and $i \in[n-1]$, let us denote by $z_{i}^{\vec{v}}$ the probability that a price drawn from $D_{i, \vec{v}_{-i}}$ does not exceed $v_{i}$, i.e.,

$$
z_{i}^{\vec{v}}=\sum_{p_{i} \in \operatorname{supp}\left(D_{i, \vec{v}_{-}}\right): p_{i} \leq v_{i}} D_{i, \vec{v}_{-i}}\left(p_{i}\right)=\sum_{v_{i}^{\prime} \in \operatorname{supp}\left(\pi_{i, \vec{v}_{-i}}\right): v_{i}^{\prime} \leq v_{i}} \frac{y_{i}^{*}\left(v_{i}^{\prime}, \vec{v}_{-i}\right)}{2},
$$


and let $X_{i}^{\vec{v}}$ denote the random variable that takes the value 1 with probability $z_{i}^{\vec{v}}$ and the value 0 with probability $1-z_{i}^{\vec{v}}$. Then the final probability in the derivation above; i.e.,

$$
\begin{gathered}
\operatorname{Pr} \quad p_{1} \sim D_{1, \vec{v}_{-1}} \quad\left[\left|\left\{j \in[n-1]: p_{j} \leq v_{j}\right\}\right|<k\right] \\
\vdots \\
p_{n-1} \sim D_{i-1, \vec{v}_{-}(n-1)}
\end{gathered}
$$

can be written as

$$
1-\operatorname{Pr}\left[\sum_{i \in[n-1]} X_{i}^{\vec{v}} \geq k\right]
$$

Let $\mu=\mathrm{E}\left[\sum_{i \in[n-1]} X_{i}^{\vec{v}}\right]$. Next, we use a Chernoff bound:

Theorem 4.14 (Chernoff Bound (as in Motwani and Raghavan (1995))). Let $X_{1}, \ldots, X_{n}$ be independent random $(0,1)$-variables such that, for $i \in[n], \operatorname{Pr}\left[X_{i}=1\right]=p_{i}$, where $p_{i} \in[0,1]$. Then, for $X=\sum_{i \in[n]} X_{i}, \mu=\mathrm{E}[X]=\sum_{i \in[n]} p_{i}$, and any $\delta>0$,

$$
\operatorname{Pr}[X \geq(1+\delta) \mu] \leq\left(\frac{e^{\delta}}{(1+\delta)^{1+\delta}}\right)^{\mu}
$$

This implies that the expression above is bounded as follows:

$$
1-\operatorname{Pr}\left[\sum_{i \in[n-1]} X_{i}^{\vec{v}} \geq\left(1+\left(\frac{k}{\mu}-1\right)\right) \mu\right] \geq 1-\left(\frac{e^{k / \mu-1}}{(k / \mu)^{k / \mu}}\right)^{\mu} .
$$

By the definition of $z_{i}^{\vec{v}}$ and the constraint in Equation (5) of the linear program, it holds that $\mu=\sum_{i \in[n-1]} z_{i}^{\vec{v}} \leq k / 2$. We can lower bound the expression above by replacing $\mu$ by $k / 2$. To see this, we first rewrite it as follows:

$$
1-\left(\frac{e^{k / \mu-1}}{(k / \mu)^{k / \mu}}\right)^{\mu}=1-\frac{e^{k-\mu+k \ln (\mu)}}{k^{k}} .
$$

The derivative of the exponent of $e$ (with respect to $\mu$ ) is positive for $\mu \in[0, k]$, which means that the exponent of $e$ is increasing in $\mu$ on $[0, k]$. Thus, replacing $\mu$ by its upper bound $k / 2$ increases the exponent and therefore decreases the expression above. Therefore:

$$
1-\operatorname{Pr}\left[\sum_{i \in[n-1]} X_{i}^{\vec{v}} \geq k\right] \geq 1-\left(\frac{e}{4}\right)^{k / 2} \geq 1-\left(\frac{e}{4}\right)^{1 / 2}=\frac{2-\sqrt{e}}{2}
$$

Continuing from Equation (7), we obtain 


$$
\begin{aligned}
\mathrm{E}_{\vec{v} \sim \pi} & {\left[\text { revenue of } M_{\pi}^{k}(\vec{v})\right] \geq \sum_{i \in[n]} \sum_{\vec{v} \in \operatorname{supp}(\pi)} \pi(\vec{v}) \sum_{p_{i} \in \operatorname{supp}\left(D_{i, \vec{v}_{-}}\right): p_{i} \leq v_{i}} p_{i} \frac{y_{i}^{*}\left(p_{i}, \vec{v}_{-i}\right)}{2} \frac{2-\sqrt{e}}{2} } \\
& =\frac{2-\sqrt{e}}{4} \sum_{i \in[n]} \sum_{\vec{v} \in \operatorname{supp}(\pi)} \pi(\vec{v}) \sum_{p_{i} \in \operatorname{supp}\left(D_{i, \vec{v}_{-i}}\right): p_{i} \leq v_{i}} p_{i} y_{i}^{*}\left(p_{i}, \vec{v}_{-i}\right) \\
& =\frac{2-\sqrt{e}}{4} \sum_{i \in[n]} \sum_{\vec{v}_{-i} \in \operatorname{supp}\left(\pi_{-i}\right)} \pi_{-i}\left(\vec{v}_{-i}\right) \sum_{v_{i}, p_{i} \in \operatorname{supp}\left(\pi_{i, \vec{v}_{-i}}\right): p_{i} \leq v_{i}} \pi_{i, \vec{v}_{-i}}\left(v_{i}\right) p_{i} y_{i}^{*}\left(p_{i}, \vec{v}_{-i}\right) \\
& =\frac{2-\sqrt{e}}{4} \sum_{i \in[n]} \sum_{\vec{v}_{-i} \in \operatorname{supp}\left(\pi_{-i}\right)} \pi_{-i}\left(\vec{v}_{-i}\right) \sum_{p_{i} \in \operatorname{supp}\left(\pi_{i, \vec{v}_{-i}}\right): p_{i} \leq v_{i}} \operatorname{Pr}_{\vec{v}_{-i} \sim \pi_{i, \vec{v}_{-i}}}\left[v_{i} \geq p_{i}\right] p_{i} y_{i}^{*}\left(p_{i}, \vec{v}_{-i}\right),
\end{aligned}
$$

which proves the claim.

Theorem 4.7 now follows by combining Lemmas 4.11 and 4.13 . We note that the approximation factor of the theorem is certainly not tight and can possibly be improved with additional work. For example, it is possible to show that for $k=1$, the revenue of $M_{\pi}^{k}$ is in fact at least $1 / 4$ of the optimal revenue. Moreover, recall that mechanism $M_{\pi}^{k}$ works by scaling the probabilities $y_{i}(\vec{v})$ down by $1 / 2$. By making this scaling factor dependent on $k$ and choosing it appropriately, we can improve the approximation factor further. We emphasize that the focus and purpose of the above result is merely to show that a constant factor of the optimal revenue (independent of the supply $k$ ) is achievable.

\section{OPEN PROBLEMS}

Besides improving any of the approximation bounds that we established in the present article, there are many other interesting further research directions. For example, it would be interesting to investigate the revenue guarantees under the additional constraint that the sequential postedprice mechanism be order oblivious: i.e., the mechanism has no control over which buyers to pick and should perform well for any possible ordering of the buyers. We are also interested in resolving some questions regarding the use of randomization in our enhanced posted-price mechanism that extracts $O(1 / d)$ of the optimal revenue: in the current proof, is it necessary to pick buyers uniformly at random, or does there exist a deterministic enhanced sequential posted-price mechanism that extracts a constant factor of the optimal revenue?

An obvious and interesting research direction is to investigate more general auction problems. In particular, to what extent can extended SPP mechanisms be applied to auctions having nonidentical items? Additionally, can such mechanisms be applied to more complex allocation constraints or specific valuation functions for the buyers? The buyers may have, for example, a demand of more than one item, or there may be a matroid feasibility constraint on the set of buyers or on the set of items that may be allocated.

\section{APPENDIX}

\section{A CONTINUOUS DISTRIBUTION PROPERTIES}

Let $\pi$ be a valuation distribution on $\left[0, a_{i}\right]^{n}$, with $a_{i} \in \mathbb{R}_{\geq 0}$ for $i \in[n]$, with density $f$ that is continuous and nowhere zero. Distribution $\pi$ is said to satisfy affiliation if and only if for every two valuation vectors $\vec{v}, \vec{w} \in \operatorname{supp}(\pi)$ it holds that $f(\vec{v} \wedge \vec{w}) f(\vec{v} \vee \vec{w}) \geq f(\vec{v}) f(\vec{w})$, where $\vec{v} \wedge \vec{w}$ is the component-wise minimum and $\vec{v} \vee \vec{w}$ is the component-wise maximum. For $i \in[n]$ and 
$\vec{v}_{-i} \in \operatorname{supp}\left(\pi_{-i}\right)$, the conditional marginal density function $f_{i}\left(\cdot \mid \vec{v}_{-i}\right)$ is defined as

$$
f_{i}\left(v_{i} \mid \vec{v}_{-i}\right)=\frac{f\left(v_{i}, \vec{v}_{-i}\right)}{\int_{0}^{a} f\left(t, \vec{v}_{-i}\right) d t},
$$

the conditional revenue curve $B_{i}\left(\cdot \mid \vec{v}_{-i}\right)$ is defined as

$$
B_{i}\left(v_{i} \mid \vec{v}_{-i}\right)=v_{i} \int_{v_{i}}^{a} f_{i}\left(t \mid \vec{v}_{-i}\right) d t,
$$

and the conditional virtual value $\phi_{i}\left(\cdot \mid \vec{v}_{-i}\right)$ is defined as

$$
\phi_{i}\left(v_{i} \mid \vec{v}_{-i}\right)=-\frac{\frac{d}{d v_{i}} B_{i}\left(v_{i} \mid \vec{v}_{-i}\right)}{f_{i}\left(v_{i} \mid \vec{v}_{-i}\right)}
$$

Denote by $F_{i}\left(\cdot \mid \vec{v}_{-i}\right)$ the cumulative distribution function corresponding to $f_{i}\left(\cdot \mid \vec{v}_{-i}\right)$. Distribution $\pi$ satisfies regularity if $\phi_{i}\left(\cdot \mid \vec{v}_{-i}\right)$ is nondecreasing for all $i \in[n]$ and $\vec{v}_{-i} \in \operatorname{supp}\left(\pi_{-i}\right)$, and it satisfies the monotone hazard rate condition if $\left(1-F_{i}\left(v_{i} \mid \vec{v}_{-i}\right)\right) /\left(f_{i}\left(v_{i} \mid \vec{v}_{-i}\right)\right)$ is nonincreasing in $v_{i}$ for all $i \in[n]$ and $\vec{v}_{-i} \in \operatorname{supp}\left(\pi_{-i}\right)$.

A discussion and justification for the above notions is outside of the scope of this article, and we refer the interested reader to Roughgarden and Talgam-Cohen (2013).

Note that Roughgarden and Talgam-Cohen (2013) proved that for any distribution $\pi$ that satisfies regularity and affiliation, the Myerson mechanism is ex-post IC, ex-post IR and optimal among all ex-post IC and ex-post IR mechanisms.

\section{B MORE REFINED ONLINE POSTED-PRICE MECHANISMS}

The revenue bound stated in Preposition 3.3 can be improved in a number of different settings. We start with the unit supply case.

Definition B.1. For a valuation distribution $\pi$ on $\mathbb{R}^{n}$, let $v_{\pi}^{\max }$ and $v_{\pi}^{\min }$ be $\max \left\{v_{i}: v \in\right.$ $\operatorname{supp}(\pi), i \in[n]\}$ and $\min \left\{\max \left\{v_{i}: i \in[n]\right\}: v \in \operatorname{supp}(\pi)\right\}$, respectively. Let $r_{\pi}^{*}=v_{\pi}^{\max } / v_{\pi}^{\min }$ be the ratio between the highest and lowest coordinate-wise maximum valuation in the support of $\pi$.

Note that $r_{\pi}^{*}$ can be much smaller than $r_{\pi}$, since the lowest coordinate-wise maximum valuation in the support of $\pi$ may be larger than the minimum valuation in the support.

Proposition B.2. Let $n \in \mathbb{N}_{\geq 1}$, and let $\pi$ be a probability distribution on $\mathbb{R}^{n}$. For the unit supply case, there exists a sequential posted-price mechanism that, when run on instance $(n, \pi, 1)$, extracts in expectation at least an $\Omega\left(1 / \log \left(r_{\pi}^{*}\right)\right)$ fraction of the optimal social welfare (and therefore also of the expected revenue of the optimal dominant strategy IC and ex-post IR auction).

Proof. The proof uses a standard bucketing trick (see, e.g., Babaioff et al. (2007)). Let $M$ be the sequential posted-price mechanism that draws a value $p$ uniformly at random from the set $S=\left\{v_{\pi}^{\min } 2^{k}: k \in\left[\left\lceil\log \left(r_{\pi}^{*}\right)-1\right\rceil\right] \cup\{0\}\right\} . M$ offers price $p$ to all the bidders in an arbitrary order, until a bidder accepts.

Let $\pi_{\max }$ be the probability distribution of the coordinate-wise maximum of $\pi$. Note that $|S|$ does not exceed $\log \left(r_{\pi_{\max }^{*}}^{*}\right)$. Therefore, the probability that $p$ is the highest possible value (among the values in $S$ ) that does not exceed the value drawn from $\pi_{\max }$ is equal to $1 / \log \left(r_{\pi}^{*}\right)$. More formally, let $\pi_{S}$ be the probability distribution from which $p$ is drawn; then

$$
\operatorname{Pr}_{v^{\max } \sim \pi_{\max }, p \sim \pi_{S}}\left[p \leq v^{\max } \cap\left(\nexists p^{\prime} \in S: p^{\prime}>p \wedge p^{\prime} \leq v^{\max }\right)\right] \leq \frac{1}{\log \left(r_{\pi}^{*}\right)} .
$$

Thus, with probability $1 / \log \left(r_{\pi}^{*}\right)$, the mechanism generates a revenue of exactly $v_{\pi}^{\min } 2^{k}$, where $k$ is the number such that the value drawn from $\pi_{\max }$ lies in between $v_{\pi}^{\min } 2^{k}$ and $v_{\pi}^{\min } 2^{k+1}$. This implies 
that with probability $1 / \log \left(r_{\pi}^{*}\right)$, the mechanism generates a revenue that lies a factor of at most $1 / 2$ away from the optimal social welfare $\operatorname{OPT}(\vec{v})$ (i.e., the coordinate-wise maximum valuation):

$$
\mathrm{E}_{\vec{v} \sim \pi}[\text { revenue of } M(\vec{v})] \geq \frac{1}{\log \left(r_{\pi}^{*}\right)} \frac{1}{2} \mathbf{E}_{\vec{v} \sim \pi}[O P T(\vec{v})] \geq \frac{1}{2 \log \left(r_{\pi}^{*}\right)} \mathbf{E}_{\vec{v} \sim \pi}[O P T(\vec{v})]
$$

The above result does not always guarantee a good revenue, for example, in the extreme case where $v_{\pi}^{\min }=0$. However, it is straightforward to generalize the above theorem such that it becomes useful for a much bigger family of probability distributions: let $\hat{v}$ and $\check{v}$ be any two values in the support of $\pi_{\max }$, and let $c(\hat{v}, \check{v})=\operatorname{Pr}_{v^{\max } \sim \pi_{\max }}\left[\check{v} \leq v^{\max } \leq \hat{v}\right]$. Then, by replacing the values $v_{\pi}^{\max }$ and $v_{\pi}^{\min }$ in the above proof by, respectively, $\hat{v}$ and $\check{v}$, we obtain a sequential posted-price mechanism that extracts in expectation a $c(\hat{v}, \check{v}) /(2 \log (\hat{v} / \check{v}))$ fraction of the optimal social welfare. By choosing $\hat{v}$ and $\check{v}$ such that this ratio is maximized, we obtain a mechanism that extracts a significant fraction of the optimal social welfare in any setting where the valuation distribution of a buyer is concentrated in a relatively not-too-large interval.

A better result can be given for the unlimited supply case.

Definition B.3. For a valuation distribution $\pi$ on $\mathbb{R}^{n}$ and any $i \in[n]$, let $v_{\pi, i}^{\max }$ and $v_{\pi, i}^{\min }$ be $\max \left\{v_{i}\right.$ : $\vec{v} \in \operatorname{supp}(\pi)\}$ and $\min \left\{v_{i}: \vec{v} \in \operatorname{supp}(\pi)\right\}$, respectively. Let $r_{\pi, i}=v_{\pi, i}^{\max } / v_{\pi, i}^{\min }$ be the ratio between the highest and lowest valuation of buyer $i$ in the support of $\pi$.

Proposition B.4. Let $n \in \mathbb{N}_{\geq 1}$, and let $\pi$ be a probability distribution on $\mathbb{R}^{n}$. There exists a sequential posted-price mechanism that, when run on instance $(n, \pi, n)$, extracts in expectation at least an $\Omega\left(1 / \log \left(\max \left\{r_{\pi, i}: i \in[n]\right\}\right)\right)$ fraction of the expected revenue of the expected optimal social welfare (and therefore also the expected revenue of the optimal dominant strategy IC and ex-post IR mechanism).

Proof. The proof applies similar techniques as those in the proof of Proposition B.2.

Let $M$ be the sequential posted-price mechanism that draws for each $i \in[n]$ a value $p_{i}$ uniformly at random from the set $S_{i}=\left\{v_{\pi, i}^{\min } 2^{k}: k \in\left[\left\lceil\log \left(r_{\pi, i}\right)-1\right\rceil\right] \cup\{0\}\right\} . M$ proposes prices to the buyers in an arbitrary order and offers price $p_{i}$ to buyer $i$.

For $i \in[n]$, let $\pi_{i}$ be the probability distribution of the $i$ th coordinate of $\pi$. Note that $\left|S_{i}\right|$ does not exceed $\log \left(r_{\pi, i}\right)$. Therefore, the probability that $p_{i}$ is the highest possible value (among the values in $S_{i}$ ) that does not exceed the value drawn from $\pi_{i}$ is at least $1 / \log \left(r_{\pi, i}\right)$. More formally, let $\pi_{S_{i}}$ be the uniform distribution on $S$; then

$$
\operatorname{Pr}_{v_{i} \sim \pi_{i}, p_{i} \sim \pi_{S_{i}}}\left[p_{i} \leq v_{i} \cap\left(\nexists p_{i}^{\prime} \in S_{i}: p_{i}^{\prime}>p_{i} \wedge p_{i}^{\prime} \leq v_{i}\right)\right] \leq \frac{1}{\log \left(r_{\pi, i}\right)} .
$$

Thus, with probability $1 / \log \left(r_{\pi, i}\right)$, the mechanism extracts from buyer $i$ a revenue of exactly $v_{i, \min } 2^{k}$, where $k$ is the number such that the value drawn from $\pi_{i}$ lies in between $v_{i, \min } 2^{i}$ and $v_{i, \min } 2^{i+1}$. This implies that with probability $1 / \log \left(r_{\pi, i}\right)$, the mechanism extracts from buyer $i$ a revenue that lies a factor of at most $1 / 2$ away from $v_{i}$. This leads to the conclusion that

$$
\begin{aligned}
\mathrm{E}_{\vec{v} \sim \pi}[\text { revenue of } M(\vec{v})] & \geq \sum_{i \in[n]} \frac{1}{\log \left(r_{\pi, i}\right)} \frac{1}{2} \mathbf{E}_{v_{i} \sim \pi_{i}}\left[v_{i}\right] \\
& \geq \frac{1}{2 \log \left(\max \left\{r_{\pi, i}: i \in[n]\right\}\right)} \sum_{i \in[n]} \mathbf{E}_{v_{i} \sim \pi_{i}}\left[v_{i}\right] \\
& =\frac{1}{2 \log \left(\max \left\{r_{\pi, i}: i \in[n]\right\}\right)} \mathbf{E}_{\vec{v} \sim \pi}[O P T(\vec{v})],
\end{aligned}
$$


where $\operatorname{OPT}(\vec{v})=\sum_{i \in[n]} v_{i}$ denotes the optimal social welfare when the buyers have valuation vector $\vec{v}$.

Clearly, the stated bound of $O\left(1 / \log \left(\max \left\{r_{\pi, i}: i \in[n]\right\}\right)\right)$ is very crude. For most practical settings, we expect that it is possible to do a much sharper revenue analysis of the mechanisms in the proofs of the above propositions, by taking the particular valuation distribution into account. Moreover, as suggested above, also for the unlimited supply case it is possible to tweak the proof in a straightforward way in order to achieve good revenue in cases where the ratios $r_{\pi, i}$ are very large. Finally, note that the mechanisms in the proofs of these two propositions do not take into account any dependence and correlation among the valuations of the buyers. When provided with a particular valuation distribution, a better revenue and sharper analysis may be obtained by taking such dependence into account and adapting the mechanisms accordingly.

The techniques used for proving these results can be applied to improve the approximation guarantees for the more general $k$-limited supply setting, for any $k \in[n]$, under special conditions. Next we give an example of such a result.

Definition B.5. For a valuation distribution $\pi$ on $\mathbb{R}^{n}$, let $v_{\pi}^{\max (k)}$ and $v_{\pi}^{\min (k)}$ be, respectively, the maximum and the minimum $k$ th largest valuation among the valuation vectors in $\operatorname{supp}(\pi)$. That is, $v_{\pi}^{\max (k)}=\max \left\{\sigma(\vec{v})_{k}: \vec{v} \in \operatorname{supp}(\pi)\right\}$ and $v_{\pi}^{\min (k)}=\min \left\{\sigma(\vec{v})_{k}: \vec{v} \in \operatorname{supp}(\pi)\right\}$, where $\sigma(\vec{v})$ is the same as $\vec{v}$, except that valuations are in nonincreasing order. Let $r_{\pi}^{(k)}=v_{\pi}^{\max (k)} / v_{\pi}^{\min (k)}$ be the ratio between these values.

Proposition B.6. Let $n \in \mathbb{N}_{\geq 1}$, and let $\pi$ be a probability distribution on $\mathbb{R}^{n}$. For any $k \in[n]$, there exists a sequential posted-price mechanism that, when run on instance $(n, \pi, k)$, extracts in expectation at least an $\Omega\left(\left(1 / \log \left(r_{\pi}^{(k)}\right)\right) \cdot\left(v_{\pi}^{\max (k)} / v_{\pi}^{\max (1)}\right)\right)$ fraction of the expected revenue of the expected optimal social welfare (and therefore also of the expected revenue of the optimal dominant strategy IC, ex-post IR mechanism).

Proof. Let $M$ be the sequential posted-price mechanism that draws a value $p$ uniformly at random from the set $S=\left\{v_{\pi}^{\min (k)} 2^{j}: j \in\left[\left\lceil\log \left(r_{\pi}^{(k)}\right)-1\right\rceil\right] \cup\{0\}\right\}$. M offers price $p$ to all the buyers in an arbitrary order, until $k$ buyers accept.

Let $\pi_{\max (k)}$ be the probability distribution of the $k$ th highest value of $\pi$. Note that $|S|$ does not exceed $\log \left(r_{\pi}^{(k)}\right)$. Therefore, the probability that $p$ is the highest possible value (among the values in $S$ ) that does not exceed the value drawn from $\pi_{\max (k)}$ is equal to $1 / \log \left(r_{\pi}^{(k)}\right)$. More formally, let $\pi_{S}$ be the probability distribution from which $p$ is drawn; then

$$
\operatorname{Pr}_{v^{\max (k)} \sim \pi_{\max (k), p \sim \pi_{S}}}\left[p \leq v^{\max (k)} \cap\left(\nexists p^{\prime} \in S: p^{\prime}>p \wedge p^{\prime} \leq v^{\max (k)}\right)\right] \leq \frac{1}{\log \left(r_{\pi}^{(k)}\right)} .
$$

Thus, with probability $1 / \log \left(r_{\pi}^{(k)}\right)$, the mechanism extracts from each winner a revenue of exactly $v_{\pi}^{\min (k)} 2^{j}$, where $j$ is the number such that the value drawn from $\pi_{\max (k)}$ lies in between $v_{\pi}^{\min (k)} 2^{j}$ and $v_{\pi}^{\min (k)} 2^{j+1}$. This implies that with probability $1 / \log \left(r_{\pi}^{(k)}\right)$, the mechanism extracts from buyer $i$ a revenue that lies a factor of $O\left(v_{\pi}^{\max (k)} / v_{\pi}^{\max (1)}\right)$ away from $v_{\pi}^{\max (1)}$. This leads to the conclusion that

$$
\mathbf{E}_{\vec{v} \sim \pi}[\text { revenue of } M(\vec{v})] \geq \Omega\left(\frac{1}{\log \left(r_{\pi}^{(k)}\right)} \cdot \frac{v_{\pi}^{\max (k)}}{v_{\pi}^{\max (1)}}\right) \sum_{i \in W_{M}} v_{\pi}^{\max (1)},
$$

where $W_{M}$ denotes the set of buyers for which mechanism $M$ allocates the service. The theorem then follows since $\sum_{i \in W_{M}} v_{\pi}^{\max (1)}=\sum_{i \in W_{O P T}} v_{\pi}^{\max (1)} \geq O P T=\sum_{i \in W_{O P T}} v_{i}$, where $W_{O P T}$ denotes 
the set of buyers at which the optimal mechanism allocates the service, and OPT is the social welfare achieved by the optimal mechanism.

We say that an instance $(n, \pi, k)$ is $k$-well-separated if for any $\vec{v} \in \operatorname{supp}(\pi)$ the $k$ th coordinatewise maximum $v_{\pi}^{\max (k)}$ is achieved only by a single buyer; i.e., the set $\left\{i: v_{i}=v_{\pi}^{\max (k)}, \vec{v} \in \operatorname{supp}(\pi)\right\}$ is a singleton. Then we can prove the following proposition.

Proposition B.7. Let $n \in \mathbb{N}_{\geq 1}$, and let $\pi$ be a discrete probability distribution on $\mathbb{R}^{n}$. For any $k \in[n]$, if the instance $(n, \pi, k)$ is $k$-well-separated, then there exists a sequential posted-price mechanism that, when run on instance $(n, \pi, k)$, extracts in expectation at least an $\Omega\left(\left(1 / \log \left(r_{\pi}^{(k)}\right)\right)\right.$. $\left.\max _{i \in[n]} \log \left(v_{\pi}^{\max (k)} / v_{\pi, i}^{\max }\right)\right)$ fraction of the expected optimal social welfare (and therefore also of the expected revenue of the optimal dominant strategy IC and ex-post IR mechanism).

Proof. Since $\pi$ is discrete, let $\delta$ be the smallest ratio larger than 1 between two valuations in $\vec{v} \in \operatorname{supp}(\pi)$, i.e., $\delta=\min _{i, j}\left\{v_{i} / v_{j}>1: \vec{v} \in \operatorname{supp}(\pi)\right\}$. Consider $\epsilon \leq \delta$ and let $M$ be the sequential posted-price mechanism that draws a value $p$ uniformly at random from the set $S=\left\{v_{\pi}^{\min (k)} \epsilon^{j}\right.$ : $\left.j \in\left[\left\lceil\log _{\epsilon}\left(r_{\pi}^{(k)}\right)-1\right\rceil\right] \cup\{0\}\right\}$. Moreover, $M$ draws for each $i \in[n]$ a value $p_{i}$ uniformly at random from the set $S_{i}=\left\{v_{\pi, i}^{\min } 2^{\ell}: \ell \in\left[\left\lfloor\log \frac{p}{v_{\pi, i}^{\min }}\right\rfloor,\left\lceil\log \frac{p}{v_{\pi, i}^{\min }}-1\right\rceil\right]\right\}$. M proposes prices to the buyers in an arbitrary order and offers price $p_{i}$ to buyer $i$.

Let $\pi_{\max (k)}$ be the probability distribution of the $k$ th coordinate-wise maximum of $\pi$. Note that $|S|$ does not exceed $\log _{\epsilon}\left(r_{\pi}^{(k)}\right)$. Therefore, the probability that $p$ is the highest possible value (among the values in $S$ ) that does not exceed the value drawn from $\pi_{\max (k)}$ is equal to $1 / \log _{\epsilon}\left(r_{\pi}^{(k)}\right)$. More formally, let $\pi_{S}$ be the probability distribution from which $p$ is drawn; then

$$
\operatorname{Pr}_{v^{\max (k)} \sim \pi_{\max (k)}, p \sim \pi_{S}}\left[p \leq v^{\max (k)} \cap\left(\nexists p^{\prime} \in S: p^{\prime}>p \wedge p^{\prime} \leq v^{\max (k)}\right)\right] \leq \frac{1}{\log _{\epsilon}\left(r_{\pi}^{(k)}\right)}
$$

Thus, with probability $1 / \log _{\epsilon}\left(r_{\pi}^{(k)}\right)$, the mechanism selects $p=v_{\pi}^{\min (k)} \epsilon^{j}$, where $j$ is the number such that the value drawn from $\pi_{\max (k)}$ lies in between $v_{\pi}^{\min (k)} \epsilon^{j}$ and $v_{\pi}^{\min (k)} \epsilon^{j+1}$. When this event occurs, since the instance is $k$-well-separated and by our choice of $\epsilon$, to the set $W_{M}$ of buyers whose valuation is at least $p$ has size exactly $k$ and corresponds of the set $W_{O P T}$ of buyers with the $k$ highest valuation in $\vec{v} \in \operatorname{supp}(\pi)$. Hence, with probability $1 / \log _{\epsilon}\left(r_{\pi}^{(k)}\right)$, mechanism $M$ extracts revenue only from buyers in $W_{O P T}$.

Now, for any $i \in W_{O P T}$, let $\pi_{i}$ be the probability distribution of the $i$ th coordinate of $\pi$. Note that $\left|S_{i}\right|$ does not exceed $\log _{\epsilon} \frac{v_{\pi, i}^{\max }}{v_{\pi}^{\max (k)}}$. Therefore, the probability that $p_{i}$ is the highest possible value (among the values in $S_{i}$ ) that does not exceed the value drawn from $\pi_{i}$ is at least $\log _{\epsilon} \frac{v_{\pi}^{\max (k)}}{v_{\pi, i}^{\max }}$. More formally, let $\pi_{S_{i}}$ be the uniform distribution on $S$; then

$$
\operatorname{Pr}_{v_{i} \sim \pi_{i}, p_{i} \sim \pi_{S_{i}}}\left[p_{i} \leq v_{i} \cap\left(\nexists p_{i}^{\prime} \in S_{i}: p_{i}^{\prime}>p_{i} \wedge p_{i}^{\prime} \leq v_{i}\right)\right] \leq \log _{\epsilon} \frac{v_{\pi}^{\max (k)}}{v_{\pi, i}^{\max }}
$$


Thus, with probability $\frac{1}{\log _{\epsilon}\left(r_{\pi}^{(k)}\right)} \cdot \log _{\epsilon} \frac{v_{\pi}^{\max (k)}}{v_{\pi, i}^{\max }}$, the mechanism extracts from buyer $i \in W_{O P T}$ a revenue of exactly $v_{i, \min } 2^{\ell}$, where $\ell$ is the number such that the value drawn from $\pi_{i}$ lies in between $v_{i, \min } 2^{\ell}$ and $v_{i, \min } 2^{\ell+1}$. This implies that with probability $\frac{1}{\log _{\epsilon}\left(r_{\pi}^{(k)}\right)} \cdot \log _{\epsilon} \frac{v_{\pi}^{\max (k)}}{v_{\pi, i}^{\max }}$, the mechanism extracts from buyer $i \in W_{O P T}$ a revenue that lies a factor of at most $1 / 2$ away from $v_{i}$. This leads to the conclusion that

$$
\begin{aligned}
\mathrm{E}_{\overrightarrow{\mathcal{v}} \sim \pi}[\text { revenue of } M(\vec{v})] & \geq \sum_{i \in W_{O P T}} \frac{1}{\log _{\epsilon}\left(r_{\pi}^{(k)}\right)} \cdot \log _{\epsilon} \frac{v_{\pi}^{\max (k)}}{v_{\pi, i}^{\max }} \frac{1}{2} \mathbf{E}_{v_{i} \sim \pi_{i}}\left[v_{i}\right] \\
& =\Omega\left(\frac{1}{\log \left(r_{\pi}^{(k)}\right)} \cdot \max _{i \in[n]} \log \frac{v_{\pi}^{\max (k)}}{v_{\pi, i}^{\max }}\right) \sum_{i \in[n]} \mathrm{E}_{v_{i} \sim \pi_{i}}\left[v_{i}\right] \\
& =\Omega\left(\frac{1}{\log \left(r_{\pi}^{(k)}\right)} \cdot \max _{i \in[n]} \log \frac{v_{\pi}^{\max (k)}}{v_{\pi, i}^{\max }}\right) \mathbf{E}_{\vec{v} \sim \pi}[O P T(\vec{v})],
\end{aligned}
$$

where $O P T(\vec{v})=\sum_{i \in W_{O P T}} v_{i}$ denotes the optimal social welfare when the buyers have valuation vector $v$.

\section{ADDRESSING SOME PRACTICAL PROBLEMS OF ENHANCED SEQUENTIAL POSTED-PRICE MECHANISMS}

A problematic aspect is that while there is no incentive for a buyer to lie, there is also no incentive to tell the truth. Therefore, incentive compatibility is only achieved in weakly dominant strategies. We note that in the literature, many (or perhaps most) truthful mechanisms are only incentive compatible in the weak sense. Such mechanisms are of theoretical interest, and may possibly be turned into more practically satisfactory mechanisms.

In the case of enhanced SPP mechanisms, the lack of a strong incentive to be truthful only applies to those buyers who are asked for their values, knowing they will not be allocated the item. Such a buyer may not cooperate at all, or may state a false value. The first problem can be resolved by compensating the buyer with some fixed small amount of money that the auctioneer obtains from the buyers who pay for the service. Having ensured some level of cooperation, how do we incentivize these buyers to be truthful?

Here is an example of such an adaptation of our enhanced SPP mechanisms that creates the proper strong incentive. Suppose now that we have provided an incentive for every buyer to reveal a valuation. At the start of the auction, using a cryptographic protocol (or just a normal sealed envelope), we ask each of the buyers for a sealed commitment of his or her value. Furthermore, for buyers being offered a price, with some (say small) probability, the buyer must reveal his or her private valuation in order to be allowed the item. If the revealed value is larger than the offered price, then the buyer is punished and the item is not allocated to him or her. Now this is strongly incentive compatible if we assume buyers are risk averse so that they will not overbid their valuation. There is clearly no monetary reason for a buyer to underbid.

\section{ON D-DIMENSIONAL DEPENDENCE VERSUS MARKOV RANDOM FIELDS OF DEGREE $D$}

This section is intended for readers who are interested in the relative generality of $d$-dimensionally dependence compared to Markov random fields of degree $d$. We assume that the reader is familiar with the definition of Markov random fields. For convenience, we will state a weaker notion here. 
Definition D.1. Given a undirected graph $G=([n], E)$, a probability distribution $\pi$ on $\mathbb{R}^{n}$ is a local Markov random field with respect to $G$ if the following property, named local Markov property, holds: for all $i \in[n], \pi_{i}$ is independent of $\pi_{[n] \backslash(\{i\} \cup \Gamma(i))}$ when conditioning on all coordinates in $\Gamma(i)$. Here, $\Gamma(i)$ denotes the neighborhood of $i$ in $G$. The degree of a Markov random field is defined as the maximum degree of a vertex in its associated graph.

(In a true Markov random field, two additional technical conditions need to be satisfied, called the pairwise Markov property and the global Markov property.) We will give an example of a 1-dimensionally dependent distribution that is not a local Markov random field with respect to any graph $G$ in which all vertices have a degree less than $(n-2) / 2$.

Consider a distribution $\pi$ on $\{0,1\}^{n+2}$. A vector $v$ drawn from $\pi$ is formed according to the following random process: we are given $2 n$ distinct probability distributions on $\{0,1\}$. We name these distributions $\pi^{i, 0}$ and $\pi^{i, 1}$, for $i \in[n]$. These distributions are such that both 0 and 1 occur with positive probability. Let $v^{\prime}$ be a value drawn from yet another distribution $\pi^{\prime}$ on $\{0,1\}$ where again both 0 and 1 have positive probability. The final generated vector is then $\left(v^{1, v^{\prime}}, v^{2, v^{\prime}}, \ldots, v^{n, v^{\prime}}, v^{\prime}, v^{\prime}\right)$, where $v^{i, v^{\prime}}$ is drawn from $\pi^{i, v^{\prime}}$.

$\pi$ is clearly 1 -dimensionally dependent, since for $i \in[n]$ the conditional marginal distribution $\pi_{i, \vec{v}_{-i}}$ is determined by only the value $v^{\prime}$, which is the value of the $(n+1)$-th coordinate. Also, the value of the $(n+1)$-th coordinate is entirely determined by the $(n+2)$-th coordinate, and vice versa.

We can also easily see that $\pi$ is not a Markov random field with respect to any graph in which all vertices have a degree less than $n / 2$. Let $G$ be a graph such that $\pi$ is a Markov random field. Suppose for contradiction that there exists an $i \in[n]$ for which it holds that $\Gamma(i) \subseteq[n]$. Then the local Markov property would be violated. Therefore, each vertex in $[n]$ is connected to either vertex $n+1$ or $n+2$. Hence, we conclude that either vertex $n+1$ or $n+2$ has at least $n / 2$ vertices attached to it.

\section{E AN ENHANCED SEQUENTIAL POSTED-PRICE MECHANISM THAT $O(1 / D)$-APPROXIMATES THE OPTIMUM REVENUE FOR MARKOV RANDOM FIELDS OF DEGREE $D$}

Consider the following procedure. Given Markov random fields of degree $d$ (in which nodes represent buyers), at each round, mark a uniformly random node, then remove the node and all its neighbors and repeat until no node is left. Note that each node is marked with probability at least $1 /(d+1)$. Consider now the following enhanced sequential posted-price mechanism: ask the unmarked buyers to reveal their valuations, and run an optimal sequential posted-price mechanism on the marked buyers, conditioned on the revealed valuations. Conditioned on the valuations of the marked buyers, the marked buyers' valuations are mutually independent. Then, one can use the results of Chawla et al. (2014) and Yan (2011) to extract a fraction of $1-1 / e$ of the optimal revenue extractable from the set of marked buyers, conditioned on the valuations of the unmarked buyers. ${ }^{9}$

\section{F AN EXAMPLE ILLUSTRATING THAT GVCG-L IS NOT A BLIND-OFFER MECHANISM}

The Generalized VCG mechanism with monopoly reserve prices (GVCG-L) is a dominant strategy IC, ex-post IR direct-revelation mechanism proposed in Chawla et al. (2014) (as a generalization of a mechanism of Li (2016) and the lookahead mechanism of Ronen (2001)), which was proved to

\footnotetext{
${ }^{9}$ We acknowledge an anonymous reviewer for pointing out this simple algorithm.
} 
extract in expectation at least half of the revenue of the maximum revenue among all ex-post IC, ex-post IR mechanisms. We show in this appendix that this mechanism is not a blind-offer mechanism. The purpose of this example is to illustrate that the restriction under which a blind-offer mechanism must make its offers (i.e., as a function of only the marginal conditional distribution of the buyer who is made the offer) is a nontrivial one, such that known approximation mechanisms cannot directly be used.

The GVCG-L mechanism is originally defined for a more general mechanism design setting than the one we study here. Restricted to our setting, the mechanism works as follows. First, let $(n, \pi, k)$ be the instance. When the buyers submit a bid vector $\vec{b}$ to the GVCG-L mechanism, it sets for each buyer a threshold price $p_{i}$. It allocates the item to a buyer $i$ if $b_{i} \geq p_{i}$ and charges to $i$ a payment of $p_{i}$. Otherwise, it does not allocate the item and charges $i$ a payment of 0 . The price $p_{i}$ is independent of $b_{i}$ and is determined as follows: Given $b_{-i}$, determine the infimum price $t_{i}$ such that $t_{i}$ would be the $k$ th highest element of the vector $\left(t_{i}, b_{-i}\right)$. Let $p_{i}$ be the price such that $p_{i} \operatorname{Pr}_{v_{i} \sim \pi_{i, b_{-}}}\left[v_{i} \geq p_{i} \mid v_{i} \geq t_{i}\right]$ is maximized. Thus, $p_{i}$ is the threshold offer that extracts the maximum expected revenue from $i$, conditioned on $i$ being among the $k$ buyers with the highest valuations.

The following is a simple example with $k=1$ and $n=2$ and independent valuations, which shows that GVCG-L is not a blind-offer mechanism. ${ }^{10}$ Let $v_{1}$ be independently drawn from the uniform distribution on $\{1,10\}$ and let $v_{2}$ be independently drawn from the uniform distribution on $\{9,11\}$. Now, GVCG-L will set $p_{2}:=9$ in case $b_{1}=v_{1}=1$, and will set $p_{2}:=11$ otherwise. Moreover, $p_{2}:=10$ if $v_{1}=9$ and $p_{2}:=11$ otherwise. It is now straightforward to see that a blind-offer mechanism cannot use this pricing scheme, since the price charged to any of the buyers may not depend on any of the bids for instances where valuations are independently drawn (since the conditional marginal distribution always equals the unconditional marginal distribution for both buyers).

In fact, it can be seen that in case of independently drawn valuations, blind-offer mechanisms are in a very strong sense equivalent to the usual sequential posted-price mechanisms.

\section{G NONINCENTIVE COMPATIBILITY OF BLIND-OFFER MECHANISMS}

Blind-offer mechanisms are not ex-post IC for a subtle reason. The price offered to a buyer $i$ by a blind-offer mechanism is entirely determined by the submitted valuations of the buyers other than $i$, and is independent of what is reported by buyer $i$ him- or herself. Also, the iteration in which a buyer is picked cannot be influenced by his or her bid. Nonetheless, incentive compatibility does not hold due to the fact that a bidder may be incentivized to misreport his or her bid in order to increase the probability of supply not running out before he or she is picked.

The following is a simple example: consider a blind-offer mechanism for two buyers, with a single unit of supply. Suppose that the mechanism always offers buyer 1 first, and then buyer 2 in case there is supply left. There are two valuation vectors in the support of the valuation distribution: $(2,2)$ and $(2,3)$. Then, in accordance with the definition of a blind-offer mechanism, the working of this mechanism may be as follows: a price of 1 is offered to buyer 1 in case the reported bid of buyer 2 is 3; a price of 3 is offered to buyer 1 in case the reported bid of buyer 2 is 2 ; in case buyer 1 rejects, a price of 1 is offered to buyer 2. It is clear that buyer 2 is now incentivized to always bid 2, even if his or her valuation is 3 . This demonstrates the nonincentive compatibility of blind-offer mechanisms.

However, blind-offer mechanisms can easily be made incentive compatible as follows: let $M$ be a non-IC blind-offer mechanism, let $\vec{b}$ be a bid vector, and let $z_{i}(\vec{b})$ be the probability that $M$ picks

\footnotetext{
${ }^{10}$ Observe that in this instance, GVCG-L is exactly the same as the lookahead mechanism. Hence, our example also shows that the lookahead mechanism is not a blind-offer mechanism.
} 
bidder $i$ before supply has run out. When a bidder is picked, we adapt $M$ by skipping that bidder with a probability $q_{i}(\vec{b})$ that is chosen in a way such that $z_{i}(\vec{b})\left(1-q_{i}(\vec{b})=\min \left\{z_{i}\left(b_{i}, \vec{b}_{-i}\right): b_{i} \in\right.\right.$ $\left.\operatorname{supp}\left(\pi_{i}\right)\right\}$. This is a blind-offer mechanism in which buyer $i$ has no incentive to lie, because now the probability that $i$ is made an offer is independent of his or her bid. Doing this iteratively for all buyers yields a dominant strategy IC mechanism $M^{\prime}$. Note that the act of skipping a bidder can be implemented by offering a price that is so high that a bidder will never accept it, and thus $M^{\prime}$ is still a blind-offer mechanism. Moreover, we note that if the probability that any bidder in $M$ is made an offer is less than a constant $c$, then in $M^{\prime}$ the probability that any bidder is offered a price is at least $c$. This fact can be used to bound the loss in revenue by a factor of $c$. Indeed, we apply this principle in the proof of Theorem 4.7 below, in order to obtain a dominant strategy IC blind-offer mechanism with a constant-factor revenue performance. ${ }^{11}$

In the main blind-offer mechanisms that we propose in Section 4.2.3, it follows from the analysis that every buyer is made an offer with a probability that is bounded from below by a constant. Applying the trick just described will result in a blind-offer mechanism that achieves a constant approximation to the optimal revenue.

Corollary G.1. There exists a dominant strategy IC blind-offer mechanism of which the expected revenue on any instance is at least a $O(1)$ fraction of the maximum expected revenue among all ex-post IC, ex-post IR mechanisms.

This corollary might be of some independent theoretical interest, since we are not aware of any other mechanisms that charge prices depending solely on the conditional marginal distributions of the buyers, and approximate the optimal revenue within a constant factor.

\section{H PROOF OF LEMMA 4.9}

Proof. Integrality for $k=n$ is the easiest to prove among these two claims, so we do that first. Note that in case $k=n$, we can safely remove the constraints in Equation (5) from the linear program, because when $k=n$, these constraints are implied by Equations (4) and (6). The linear program that remains tells us how to optimize a sum of convex combinations of values. That is, it effectively tells us to pick for each $i \in[n]$ and $\vec{v}_{-i} \in \operatorname{supp}\left(\pi_{-i}\right)$ a convex combination of the values $\left\{v_{i} \operatorname{Pr}_{v_{i}^{\prime} \sim \pi_{i, \vec{v}_{-i}}}\left[v_{i}^{\prime} \geq v_{i}\right]\right\}_{v_{i}^{\prime} \in \operatorname{supp}\left(\pi_{i, \vec{v}_{-i}}\right)}$. The optimal solution is therefore to put weight 1 on the maximum values in these sets, i.e., to set $y_{i}\left(v_{i}, \vec{v}_{-i}\right)$ to 1 for the value $v_{i}$ that maximizes $v_{i} \operatorname{Pr}_{v_{i}^{\prime} \sim \pi_{i, \vec{v}_{-i}}}\left[v_{i}^{\prime} \geq v_{i}\right]$

It remains to prove the first claim. To this aim, let us first introduce some specialized notation: let $\sigma$ now be a probability distribution on a finite subset of $\mathbb{R}_{\geq 0}$ and $x \in \operatorname{supp}(\sigma)$; we write $\operatorname{prec}_{\sigma}(x)$ to denote $\max \operatorname{supp}(\sigma) \cap[0, x)$ if $\operatorname{supp}(\sigma) \cap[0, x)$ is nonempty. Otherwise, if $\operatorname{supp}(\sigma) \cap[0, x)=\varnothing$, we define $\operatorname{prec}_{\sigma}(x)=0$. Similarly, we write $\operatorname{succ}_{\sigma}(x)$ to denote $\min \operatorname{supp}(\sigma) \cap(x, \infty]$. (We leave $\operatorname{succ}_{\sigma}(x)$ undefined if $\operatorname{supp}(\sigma) \cap(x, \infty]$ is empty. $)$

Suppose now that $A$ is an optimal ex-post IC, ex-post IR mechanism. For $\vec{v} \in \operatorname{supp}(\pi)$, denote by $x(\vec{v})$ the expected allocation vector output by $A$ when the buyers report valuation vector $\vec{v}$ (so that for $i \in[n]$, the value $x_{i}(\vec{v})$ is the probability that $i$ gets allocated service, when the buyers report $\vec{v}$ ) and denote by $p(\vec{v})$ the vector of expected prices charged by $A$ when the buyers report $\vec{v}$. Ex-post incentive compatibility states that

$$
\forall i \in[n], \vec{v}_{-i} \in \operatorname{supp}\left(\pi_{-i}\right),\left(v_{i}, v_{i}^{\prime}\right) \in \operatorname{supp}\left(\pi_{i}\right)^{2}: v_{i} x_{i}\left(v_{i}, \vec{v}_{-i}\right)-p_{i}\left(v_{i}, \vec{v}_{-i}\right) \geq v_{i} x_{i}\left(v_{i}^{\prime}, \vec{v}_{-i}\right)-p_{i}\left(v_{i}^{\prime}, \vec{v}_{-i}\right)
$$

\footnotetext{
${ }^{11}$ More precisely, for the particular (nontruthful) blind-offer mechanism that we propose and analyze in Section 4.2.3, it will turn out that applying the transformation described here does not result in any additional loss in revenue.
} 
and ex-post individual rationality states that

$$
\forall i \in[n], \vec{v} \in \operatorname{supp}(\pi): v_{i} x_{i}\left(v_{i}, \vec{v}_{-i}\right)-p_{i}\left(v_{i}, \vec{v}_{-i}\right) \geq 0 .
$$

The next lemma states that, in $A$, the allocation probability for a buyer is nondecreasing in his or her reported valuation.

Lemma H.1. For all $i \in[n]$, all $\vec{v}_{-i} \in \pi_{-i}$, and all $v_{i}, v_{i}^{\prime} \in \operatorname{supp}\left(\pi_{i, \vec{v}_{-i}}\right)$, with $v_{i}<v_{i}^{\prime}$, it holds that

$$
x_{i}\left(v_{i}, \vec{v}_{-i}\right) \leq x_{i}\left(v_{i}^{\prime}, \vec{v}_{-i}\right) .
$$

Proof. By way of contradiction, we assume that $\epsilon=x_{i}\left(v_{i}, \vec{v}_{-i}\right)-x_{i}\left(v_{i}^{\prime}, \vec{v}_{-i}\right)>0$. By ex-post incentive compatibility, it holds that

$$
\begin{aligned}
& v_{i} x_{i}\left(v_{i}, \vec{v}_{-i}\right)-p_{i}\left(v_{i}, \vec{v}_{-i}\right) \geq v_{i} x_{i}\left(v_{i}^{\prime}, \vec{v}_{-i}\right)-p_{i}\left(v_{i}^{\prime}, \vec{v}_{-i}\right), \\
& v_{i}^{\prime} x_{i}\left(v_{i}^{\prime}, \vec{v}_{-i}\right)-p_{i}\left(v_{i}^{\prime}, \vec{v}_{-i}\right) \geq v_{i}^{\prime} x_{i}\left(v_{i}, \vec{v}_{-i}\right)-p_{i}\left(v_{i}, \vec{v}_{-i}\right)
\end{aligned}
$$

We now rewrite these inequalities as

$$
\begin{aligned}
& v_{i} x_{i}\left(v_{i}, \vec{v}_{-i}\right)-v_{i} x_{i}\left(v_{i}^{\prime}, \vec{v}_{-i}\right) \geq p_{i}\left(v_{i}, \vec{v}_{-i}\right)-p_{i}\left(v_{i}^{\prime}, \vec{v}_{-i}\right), \\
& v_{i}^{\prime} x_{i}\left(v_{i}, \vec{v}_{-i}\right)-v_{i}^{\prime} x_{i}\left(v_{i}^{\prime}, \vec{v}_{-i}\right) \leq p_{i}\left(v_{i}, \vec{v}_{-i}\right)-p_{i}\left(v_{i}^{\prime}, \vec{v}_{-i}\right)
\end{aligned}
$$

This results in the following pair of inequalities:

$$
\begin{aligned}
& v_{i} \epsilon \geq p_{i}\left(v_{i}, \vec{v}_{-i}\right)-p_{i}\left(v_{i}^{\prime}, \vec{v}_{-i}\right), \\
& v_{i}^{\prime} \epsilon \leq p_{i}\left(v_{i}, \vec{v}_{-i}\right)-p_{i}\left(v_{i}^{\prime}, \vec{v}_{-i}\right) .
\end{aligned}
$$

The two inequalities contradict each other, because $v_{i}^{\prime}>v_{i}$ and we assumed $\epsilon>0$.

The next lemma upper bounds the prices charged by $A$.

Lemma H.2. For all $i \in[n]$, all $\vec{v}_{-i} \in \operatorname{supp}\left(\vec{v}_{-i}\right)$, and all $v_{i} \in \operatorname{supp}\left(\pi_{i, \vec{v}_{-i}}\right)$, it holds that

$$
p_{i}\left(v_{i}, \vec{v}_{-i}\right) \leq v_{i} x_{i}\left(v_{i}, \vec{v}_{-i}\right)-\sum_{v_{i}^{\prime} \in \sup p\left(\pi_{i, \vec{v}_{-i}}\right): v_{i}^{\prime}<v_{i}}\left(\operatorname{succ}_{\pi_{i, \vec{v}_{-i}}}\left(v_{i}\right)-v_{i}\right) x_{i}\left(v_{i}^{\prime}, \vec{v}_{-i}\right) .
$$

Proof. For $v_{i}^{\prime} \in \operatorname{supp}\left(\pi_{i, \vec{v}_{-i}}\right)$, the ex-post IC constraint for $\vec{v}_{-i}, v_{i}^{\prime}, \operatorname{prec}_{\pi_{i, \vec{v}_{-i}}}\left(v_{i}\right)$ can be written as

$$
v_{i}^{\prime}\left(x_{i}\left(v_{i}^{\prime}, \vec{v}_{-i}\right)-x_{i}\left(v_{i}^{\prime \prime}, \vec{v}_{-i}\right)\right) \geq p_{i}\left(v_{i}^{\prime}, \vec{v}_{-i}\right)-p_{i}\left(v_{i}^{\prime \prime}, \vec{v}_{-i}\right),
$$

where $v_{i}^{\prime \prime}=\operatorname{prec}_{\pi_{i, \vec{v}_{-}}}\left(v_{i}\right)$. Summing the above over all $v_{i}^{\prime} \in \operatorname{supp}\left(\pi_{i, \vec{v}_{-i}}\right), v_{i}^{\prime}<v_{i}$ yields Equation (9).

The optimal revenue among all ex-post IC, ex-post IR mechanisms (and thus the expected revenue of $A$ ) can be written as the following linear program, where $(x(\vec{v}))_{\vec{v} \in \operatorname{supp}(\pi)}$ and $(p(\vec{v}))_{\vec{v} \in \operatorname{supp}(\pi)}$ are the variables:

$$
\begin{aligned}
& \max \left\{\sum_{i \in[n]} \sum_{\vec{v} \in \operatorname{supp}(\pi)} p_{i}(\vec{v}) \pi(\vec{v}) \mid\right. \\
& \forall i \in[n], \vec{v} \in \operatorname{supp}(\pi): v_{i} x_{i}(\vec{v})-p_{i}(\vec{v}) \geq 0 \\
& \forall i \in[n],\left(v_{i}, v_{i}^{\prime}\right) \in \operatorname{supp}\left(\pi_{i}\right)^{2}, \vec{v}_{-i} \in \operatorname{supp}\left(\pi_{-i, v_{i}}\right): \\
& \quad v_{i} x_{i}\left(v_{i}, \vec{v}_{-i}\right)-p_{i}\left(v_{i}, \vec{v}_{-i}\right) \geq v_{i} x_{i}\left(v_{i}^{\prime}, \vec{v}_{-i}\right)-p_{i}\left(v_{i}^{\prime}, \vec{v}_{-i}\right) \\
& \forall \vec{v} \in \operatorname{supp}(\pi): \sum_{i} x_{i}(\vec{v}) \leq k \\
& \left.\forall i \in[n], \vec{v} \in \operatorname{supp}(\pi): 0 \leq x_{i}(\vec{v}) \leq 1\right\} .
\end{aligned}
$$


In the above linear program, Equation (11) contains the ex-post IR constraints, Equation(12) contains the ex-post IC constraints, and Equation (13) expresses that the service cannot be provided to more than $k$ buyers.

By Lemma H.1, it is possible to add to the above linear program the constraints $x_{i}\left(v_{i}, \vec{v}_{-i}\right) \geq$ $x_{i}\left(\operatorname{prec}_{\pi_{i, \vec{v}_{-}}}\left(v_{i}\right)\right)$ for $i \in[n], \vec{v}_{-i} \in \operatorname{supp}\left(\pi_{-i}\right), v_{i} \in \operatorname{supp}\left(\pi_{i, \vec{v}_{-i}}\right)$. Moreover, by Lemma H.2, we can replace the objective function by

$$
\sum_{i \in[n]} \sum_{\vec{v} \in \operatorname{supp}(\pi)} \pi(\vec{v})\left(v_{i} x_{i}\left(v_{i}, \vec{v}_{-i}\right)-\sum_{v_{i}^{\prime} \in \operatorname{supp}\left(\pi_{i, \vec{v}_{-i}}\right): v_{i}^{\prime}<v_{i}}\left(\operatorname{succ}_{\pi_{i, \vec{v}_{-i}}}\left(v_{i}\right)-v_{i}\right) x_{i}\left(v_{i}^{\prime}, \vec{v}_{-i}\right)\right)
$$

and remove the constraints in Equations (11) and (12). This results in the following linear program that upper bounds the optimal revenue among the ex-post IC, ex-post IR mechanisms:

$$
\begin{gathered}
\max \left\{\sum_{i \in[n]} \sum_{\vec{v} \in \operatorname{supp}(\pi)} \pi(\vec{v})\left(v_{i} x_{i}\left(v_{i}, \vec{v}_{-i}\right)-\sum_{v_{i}^{\prime} \in \operatorname{supp}\left(\pi_{i, \vec{v}_{-i}}\right): v_{i}^{\prime}<v_{i}}\left(\operatorname{succ}_{\pi_{i, \vec{v}_{-i}}}\left(v_{i}\right)-v_{i}\right) x_{i}\left(v_{i}^{\prime}, \vec{v}_{-i}\right)\right)\right. \\
\forall i \in[n], \vec{v} \in \operatorname{supp}(\pi): x_{i}(\vec{v}) \geq x_{i}\left(\operatorname{prec}_{\pi_{i, \vec{v}_{-i}}}\left(v_{i}\right), \vec{v}_{-i}\right) \\
\forall \vec{v} \in \operatorname{supp}(\pi): \sum_{i} x_{i}(\vec{v}) \leq k \\
\left.\forall i \in[n], \vec{v} \in \operatorname{supp}(\pi): 0 \leq x_{i}(\vec{v}) \leq 1\right\} .
\end{gathered}
$$

We will show that the linear program (Equations (3) to (6)) is equivalent to the above. Set $y_{i}(\vec{v})=x_{i}(\vec{v})-x_{i}\left(\operatorname{prec}_{\pi_{i, \vec{v}_{-i}}}\left(v_{i}\right), \vec{v}_{-i}\right)$ for all $i \in[n], \vec{v} \in \operatorname{supp}(\pi)$, and observe that the constraints in Equations (16), (17), and (18) are then equivalent to Equations (4), (5), and (6), respectively. Moreover, with this correspondence between $y$ and $x$, we can rewrite the objective function as follows:

$$
\begin{aligned}
& \sum_{i \in[n]} \sum_{\vec{v} \in \operatorname{supp}(\pi)} \pi(\vec{v})\left(v_{i} x_{i}\left(v_{i}, \vec{v}_{-i}\right)-\sum_{v_{i}^{\prime} \in \operatorname{supp}\left(\pi_{i, \vec{v}_{-i}}\right): v_{i}^{\prime}<v_{i}}\left(\operatorname{succ}_{\pi_{i, \vec{v}_{-i}}}\left(v_{i}\right)-v_{i}\right) x_{i}\left(v_{i}^{\prime}, \vec{v}_{-i}\right)\right) \\
& =\sum_{i \in[n]} \sum_{\vec{v}_{-i} \in \operatorname{supp}\left(\pi_{-i}\right)} \pi_{-i}\left(\vec{v}_{-i}\right) \sum_{v_{i} \in \operatorname{supp}\left(\pi_{i, \vec{v}_{-i}}\right)} \pi_{i, \vec{v}_{-i}}\left(v_{i}\right) v_{i} x_{i}\left(v_{i}, \vec{v}_{-i}\right) \\
& -\sum_{i \in[n]} \sum_{\vec{v}_{-i} \in \operatorname{supp}\left(\pi_{-i}\right)} \pi_{-i}\left(\vec{v}_{-i}\right) \sum_{v_{i} \in \operatorname{supp}\left(\pi_{i, \vec{v}_{-i}}\right)} \pi_{i, \vec{v}_{-i}}\left(v_{i}\right) . \\
& \sum_{v_{i}^{\prime} \in \operatorname{supp}\left(\pi_{i, \vec{v}_{-i}}\right): v_{i}^{\prime}<v_{i}}\left(\operatorname{succ}_{\pi_{i, \vec{v}_{-i}}}\left(v_{i}\right)-v_{i}\right) x_{i}\left(v_{i}^{\prime}, \vec{v}_{-i}\right) \\
& =\sum_{i \in[n]} \sum_{\vec{v}_{-i} \in \operatorname{supp}\left(\pi_{-i}\right)} \pi_{-i}\left(\vec{v}_{-i}\right) \sum_{v_{i} \in \operatorname{supp}\left(\pi_{i, \vec{v}_{-i}}\right)} \pi_{i, \vec{v}_{-i}}\left(v_{i}\right) v_{i} x_{i}\left(v_{i}, \vec{v}_{-i}\right) \\
& -\sum_{i \in[n]} \sum_{\vec{v}_{-i} \in \operatorname{supp}\left(\pi_{-i}\right)} \pi_{-i}\left(\vec{v}_{-i}\right) \sum_{v_{i} \in \operatorname{supp}\left(\pi_{i, \vec{v}_{-i}}\right)} x_{i}\left(v_{i}, \vec{v}_{-i}\right)\left(\operatorname{succ}_{\pi_{i, \vec{v}_{-i}}}\left(v_{i}\right)-v_{i}\right) \operatorname{Pr}_{v_{i}^{\prime} \sim \pi_{i, \vec{v}_{-i}}}\left[v_{i}^{\prime}>v_{i}\right] \\
& =\sum_{i \in[n]} \sum_{\vec{v}_{-i} \in \operatorname{supp}\left(\pi_{-i}\right)} \pi_{-i}\left(\vec{v}_{-i}\right) \sum_{v_{i} \in \operatorname{supp}\left(\pi_{i, \vec{v}_{-i}}\right)} x_{i}\left(v_{i}, \vec{v}_{-i}\right) .
\end{aligned}
$$




$$
\begin{aligned}
& \left(\pi_{i, \vec{v}_{-i}}\left(v_{i}\right) v_{i}-\left(\operatorname{succ}_{\pi_{i, \vec{v}_{-i}}}\left(v_{i}\right)-v_{i}\right) \operatorname{Pr}_{v_{i}^{\prime} \sim \pi_{i, \vec{v}_{-i}}}\left[v_{i}^{\prime}>v_{i}\right]\right) \\
& =\sum_{i \in[n]} \sum_{\vec{v}_{-i} \in \operatorname{supp}\left(\pi_{-i}\right)} \pi_{-i}\left(\vec{v}_{-i}\right) \sum_{v_{i} \in \operatorname{supp}\left(\pi_{i, \vec{v}_{-i}}\right)} x_{i}\left(v_{i}, \vec{v}_{-i}\right) . \\
& \left(\operatorname{Pr}_{v_{i}^{\prime} \sim \pi_{i, \vec{v}_{-i}}}\left[v_{i}^{\prime} \geq v_{i}\right] v_{i}-\left(\operatorname{succ}_{\pi_{i, \vec{v}_{-i}}}\left(v_{i}\right)\right) \operatorname{Pr}_{v_{i}^{\prime} \sim \pi_{i, \vec{v}_{-i}}}\left[v_{i}^{\prime}>v_{i}\right]\right) \\
& =\sum_{i \in[n]} \sum_{\vec{v}_{-i} \in \operatorname{supp}\left(\pi_{-i}\right)} \pi_{-i}\left(\vec{v}_{-i}\right) \sum_{v_{i} \in \operatorname{supp}\left(\pi_{i, \vec{v}_{-i}}\right)}\left(x_{i}\left(v_{i}, \vec{v}_{-i}\right)-x_{i}\left(\operatorname{prec}_{\pi_{i, \vec{v}_{-i}}}\left(v_{i}\right), \vec{v}_{-i}\right)\right) v_{i} \operatorname{Pr}_{v_{i}^{\prime} \sim \pi_{i, \vec{v}_{-i}}}\left[v_{i}^{\prime} \geq v_{i}\right] \\
& =\sum_{i \in[n]} \sum_{\vec{v}_{-i} \in \operatorname{supp}\left(\pi_{-i}\right)} \pi_{-i}\left(\vec{v}_{-i}\right) \sum_{v_{i} \in \operatorname{supp}\left(\pi_{i, \vec{v}_{-i}}\right)} \operatorname{Pr}_{v_{i}^{\prime} \sim \pi_{i, \vec{v}_{-i}}}\left[v_{i}^{\prime} \geq v_{i}\right] v_{i} y_{i}\left(v_{i}, \vec{v}_{-i}\right) .
\end{aligned}
$$

This completes the proof.

\section{ACKNOWLEDGMENTS}

Part of the work has been done when Marek Adamczyk, Allan Borodin, Diodato Ferraioli, and Bart de Keijzer were all at Sapienza University of Rome. A shorter conference version of this article appeared as (Adamczyk et al. 2015). We thank Joanna Drummond, Brendan Lucier, Tim Roughgarden and anonymous conference and journal referees for their many constructive comments which we have incorporated.

\section{REFERENCES}

I. Abraham, S. Athey, M. Babaioff, and M. Grubb. 2013. Peaches, lemons, and cookies: Designing auction markets with dispersed information. In Proceedings of the 14th ACM Conference on Electronic Commerce. ACM, 7-8.

M. Adamczyk, A. Borodin, D. Ferraioli, B. de Keijzer, and S. Leonardi. 2015. Sequential posted price mechanisms with correlated valuations. In Proceedings of the 11th International Conference on Web and Internet Economics (WINE'15). Springer, $1-15$.

M. Adamczyk, M. Sviridenko, and J. Ward. 2014. Submodular stochastic probing on matroids. In Proceedings of the 31st International Symposium on Theoretical Aspects of Computer Science (STACS'14). Schloss Dagstuhl, 29-40.

L. M. Ausubel. 1999. A Generalized Vickrey Auction. Technical Report. University of Maryland.

M. Babaioff, S. Dughmi, R. Kleinberg, and A. Slivkins. 2012. Dynamic pricing with limited supply. In Proceedings of the 13th ACM Conference on Economics and Computation (EC'12). ACM, 74-91.

M. Babaioff, N. Immorlica, and R. Kleinberg. 2007. Matroids, secretary problems, and online mechanisms. In Proceedings of the 18th ACM-SIAM Symposium on Discrete Algorithms (SODA'07). SIAM.

M. Babaioff, N. Immorlica, B. Lucier, and S. M. Weinberg. 2014. A simple and approximately optimal mechanism for an additive buyer. In Proceedings of the 55th Annual IEEE Symposium on Foundations of Computer Science (FOCS'14). IEEE, 21-30.

M. Babaioff, R. Kleinberg, and R. Paes Leme. 2012. Optimal mechanisms for selling information. In Proceedings of the 13th ACM Conference on Electronic Commerce. ACM, 92-109.

A. Blum and J. D. Hartline. 2005. Near-optimal online auctions. In Proceedings of the 16th ACM-SIAM Symposium on Discrete Algorithms (SODA'05). SIAM, 1156-1163.

A. Blum, V. Kumar, A. Rudra, and F. Wu. 2004. Online learning in online auctions. Theoretical Computer Science 324 (2004), 137-146.

L. Blumrosen and T. Holenstein. 2008. Posted prices vs. negotiations: An asymptotic analysis. In Proceedings of the 9th ACM Conference on Economics and Computation (EC'08). ACM, 49-49.

T. Börgers. 2015. An Introduction to the Theory of Mechanism Design. Oxford University Press.

S. Chawla, H. Fu, and A. Karlin. 2014. Approximate revenue maximization in interdependent value settings. In Proceedings of the 15th ACM Conference on Economics and Computation (EC'14). ACM, 277-294.

S. Chawla, J. D. Hartline, D. L. Malec, and B. Sivan. 2010. Multi-parameter mechanism design and sequential posted pricing. In Proceedings of the 42nd ACM Symposium on Theory of Computing (STOC'10). ACM, 311-320.

N. Chen, N. Immorlica, A. R. Karlin, M. Mahdian, and A. Rudra. 2009. Approximating matches made in heaven. In Proceedings of the 36th International Colloquium on Automata, Languages and Programming (ICALP'09). Springer, 266-278.

E. H. Clarke. 1971. Multipart pricing of public goods. Public Choice 11, 1 (1971), 17-33.

J. Cremer and R. P. McLean. 1988. Full extraction of the surplus in Bayesian and dominant strategy auctions. Econometrica 56, 6 (1988), 1247-1257. 
N. R. Devanur, J. Morgenstern, V. Syrgkanis, and S. M. Weinberg. 2015. Simple auctions with simple strategies. In Proceedings of the 16th ACM Conference on Economics and Computation (EC'15). ACM, 305-322.

S. Dobzinski, H. Fu, and R. D. Kleinberg. 2011. Optimal auctions with correlated bidders are easy. In Proceedings of the 43rd ACM Symposium on Theory of Computing (STOC'11). ACM, 129-138.

R. El-Yaniv, A. Fiat, R. M. Karp, and G. Turpin. 2001. Optimal search and one-way trading online algorithms. Algorithmica 30, 1 (2001), 101-139.

M. Feldman, N. Gravin, and B. Lucier. 2015. Combinatorial auctions via posted prices. In Proceedings of the 26th ACM-SIAM Symposium on Discrete Algorithms (SODA'15). SIAM, 123-135.

M. Feldman, O. Svensson, and R. Zenklusen. 2016. Online contention resolution schemes. In Proceedings of the 27th ACMSIAM Symposium on Discrete Algorithms. SIAM, 1014-1033.

T. Groves. 1973. Incentives in teams. Econometrica 14, 4 (1973), 617-631.

A. Gupta and V. Nagarajan. 2013. A stochastic probing problem with applications. In Proceedings of the 16th Conference on Integer Programming and Combinatorial Optimization (IPCO'13). Springer.

S. Hart and N. Nisan. 2012. Approximate revenue maximization with multiple items. In Proceedings of the 13th ACM Conference on Economics and Computation (EC'12). ACM, 656-656.

J. D. Hartline and T. Roughgarden. 2009. Simple versus optimal mechanisms. SIGecom Exchanges 8, 1 (2009), 5:1-5:3.

J. H. Kagel, R. M. Harstad, and D. Levin. 1987. Information impact and allocation rules in auctions with affiliated private values: A laboratory study. Econometrica 55, 6 (1987), 1275-1304.

R. Kleinberg and T. Leighton. 2003. The value of knowing a demand curve: Bounds on regret for online posted-price auctions. In Proceedings of the 44th Annual IEEE Symposium on Foundations of Computer Science (FOCS'03). IEEE, 594-594.

R. Kleinberg and S. M. Weinberg. 2012. Matroid prophet inequalities. In Proceedings of the 44th ACM Symposium on Theory of Computing (STOC'12). ACM, 123-136.

R. Lavi and N. Nisan. 2004. Competitive analysis of incentive compatible on-line auctions. Theoretical Computer Science 310, 1-3 (2004), 159-180.

Shengwu Li. 2015. Obviously Strategy-Proof Mechanisms. Economic Review, forthcoming.

Y. Li. 2016. Approximation in mechanism design with interdependent values. Games and Economic Behavior 103, Supplement C (2016), 225-253.

E. Maskin and P. Dasgupta. 2000. Efficient auctions. Quarterly fournal of Economics 115 (2000), 341-388.

R. P. McAfee, J. McMillan, and P. J. Reny. 1989. Extracting the surplus in the common-value auction. Econometrica 57, 6 (1989), 1451-1459.

R. P. McAfee and P. J. Reny. 1992. Correlated information and mechanism design. Econometrica 60, 2 (1992), 395-421.

R. Motwani and P. Raghavan. 1995. Randomized Algorithms. Cambridge University Press.

R. B. Myerson. 1981. Optimal auction design. Mathematics of Operations Research 6, 1 (1981), $58-73$.

C. H. Papadimitriou and G. Pierrakos. 2011. On optimal single-item auctions. In Proceedings of the 34rd Annual ACM Symposium on Theory of Computing. ACM, 119-128.

A. Ronen. 2001. On approximating optimal auctions. In Proceedings of the 2nd ACM Conference on Economics and Computation (EC'01). ACM, 11-17.

A. Ronen and A. Saberi. 2002. On the hardness of optimal auctions. In Proceedings of the 43rd Annual IEEE Symposium on Foundations of Computer Science. IEEE, 396-405.

T. Roughgarden and I. Talgam-Cohen. 2013. Optimal and near-optimal mechanism design with interdependent values. In Proceedings of the 13th ACM Conference on Economics and Computation (EC'13). ACM, 767-784.

A. Rubinstein and S. M. Weinberg. 2015. Simple mechanisms for a subadditive buyer and applications to revenue monotonicity. In Proceedings of the 16th ACM Conference on Economics and Computation (EC'15). ACM, 377-394.

T. Sandholm and A. Gilpin. 2004. Sequences of take-it-or-leave-it offers: Near-optimal auctions without full valuation revelation. In Agent-Mediated Electronic Commerce V. Springer.

I. Segal. 2003. Optimal pricing mechanisms with unknown demand. American Economic Review 93, 3 (2003), 509-529.

V. Syrgkanis, D. Kempe, and E. Tardos. 2015. Information asymmetries in common-value auctions with discrete signals. In Proceedings of the 16th ACM Conference on Economics and Computation. ACM, 303-303.

W. Vickrey. 1961. Counterspeculation, auctions, and competitive sealed tenders. fournal of Finance 16, 1 (1961), 8-37.

Q. Yan. 2011. Mechanism design via correlation gap. In Proceedings of the 22nd ACM-SIAM Symposium on Discrete Algorithms (SODA'11). SIAM, 710-719.

Received July 2016; revised April 2017; accepted September 2017 